\title{
Ultrastrong plasmon-phonon coupling via epsilon-near-zero nanocavities
}

\author{
Daehan Yoo ${ }^{1,+}$, Fernando de León-Pérez ${ }^{2,3,+}$, Matthew Pelton ${ }^{4}$, \\ In-Ho Lee ${ }^{1}$, Daniel A. Mohr ${ }^{1}$, Markus B. Raschke ${ }^{5}$, \\ Joshua D. Caldwell ${ }^{6}$, Luis Martín-Moreno ${ }^{3, *}$, and Sang-Hyun $\mathrm{Oh}^{1, *}$
}

${ }^{1}$ Department of Electrical and Computer Engineering, University of Minnesota, 200 Union St. S.E., Minneapolis, MN, USA

${ }^{2}$ Centro Universitario de la Defensa de Zaragoza, Ctra. de Huesca s/n, Zaragoza, Spain

${ }^{3}$ Instituto de Nanociencia y Materiales de Aragón (INMA) and Departamento de Física de la Materia Condensada, CSIC-Universidad de Zaragoza, Zaragoza, Spain

${ }^{4}$ Department of Physics, University of Maryland, Baltimore County, Baltimore, MD, USA

${ }^{5}$ Department of Physics and JILA, University of Colorado, Boulder, CO, USA

${ }^{6}$ Department of Mechanical Engineering, Vanderbilt University, Nashville, TN, USA

*E-mail: Imm@unizar.es and sang@umn.edu

+these authors contributed equally to this work

\begin{abstract}
Vibrational ultrastrong coupling (USC), where the light-matter coupling strength is comparable to the vibrational frequency of molecules, presents new opportunities to probe the interactions of molecules with zero-point fluctuations, harness cavitymodified chemical reactions, and develop novel devices in the mid-infrared spectral range. Here we use epsilon-near-zero nanocavities filled with a model polar medium $\left(\mathrm{SiO}_{2}\right)$ to demonstrate USC between phonons and gap plasmons. We present classical and quantum mechanical models to quantitatively describe the observed plasmon-phonon USC phenomena and demonstrate a modal splitting of up to $50 \%$ of the resonant frequency (normalized coupling strength $\eta>0.25$ ). Our wafer-scale nanocavity platform will enable a broad range of vibrational transitions to be harnessed for USC applications.
\end{abstract}

The field of cavity quantum electrodynamics (QED) has generally divided light-matter interactions into two regimes: weak coupling (WC), when losses exceed the light-matter coupling strength, and strong coupling (SC), when the coupling strength dominates $^{1,2}$. For weak-coupling phenomena such as the Purcell effect ${ }^{3}$, Fano interference ${ }^{4}$, and surface-enhanced infrared absorption $^{5}$, the coupled systems exchange energy on a time scale slower than the decay rates. In contrast, within the SC regime, the oscillators exchange their energy reversibly and coherently over an extended time frame that is longer than the decay rates ${ }^{6-14}$, enabling applications such as quantum information processing ${ }^{15}$. Furthermore, strong coupling leads to the formation of hybridized energy states that are different from those of the bare constituents, leading to novel phenomena such as the modification of chemical reaction rates ${ }^{2,16,17}$ and of ground-state reactivity ${ }^{18}$.

Even more exotic phenomena can occur when the normalized coupling strength, $\eta$, of the system exceeds $\sim 0.1$, where $\eta$ is defined as the ratio of the light-matter coupling strength, $g$, to the mid-gap frequency, $\omega$. In this ultrastrong coupling (USC) regime ${ }^{1,2,19,20}$, some of the standard approximations that are valid for WC and SC, such as the rotating-wave approximation, are expected to break down. Furthermore, transitioning from SC to USC implies that the hybrid mode exhibits substantially more oscillations between light and matter states prior to decay, and such fast and efficient interactions can enable novel ultrafast devices ${ }^{19,21}$. Another striking phenomenon predicted in the USC regime, resulting from the antiresonant term in the light-matter coupling equation, is the possibility to extract virtual photons from the modified ground state via a dynamic Casimir effect $^{22,23}$. Finally, USC between light and molecules has the potential to modify or enhance chemical reactions beyond what is possible in the SC regime ${ }^{19}$.

USC has been demonstrated using photochromic molecules ${ }^{24}$, circuit QED systems ${ }^{25}$, intersubband polaritons ${ }^{26}$, molecular liquids $^{27}$, and two-dimensional electron gase ${ }^{28}$. SC to vibrational modes at mid-infrared (MIR) frequencies has been demonstrated in various systems ${ }^{18,29-32}$, enabling applications such as surface-enhanced vibrational spectroscopy $y^{5,33}$, thermal emission and signature control, and modified heat transfer. It has been challenging, however, to achieve USC at MIR frequencies, particularly in solid-state systems, because of the relatively weak oscillator strengths for vibrational modes originating from lattice ionic motions. Previous demonstrations have involved extended microcavity structures ${ }^{2,18,27}$, opening up a new physical regime at these technologically important frequencies, but with limited possibilities for novel nonlinear effects. 
Here we demonstrate vibrational USC within nanocavities, drastically reducing the size of the system and thus the amount of material involved in achieving MIR USC. Specifically, we use coaxial nanocavities, which exhibit strong transmission resonances and field enhancements close to the cutoff frequency of the $\mathrm{TE}_{11}$-like mode; these can be understood as resulting from excitation of the zeroth-order Fabry-Perot resonance ${ }^{34}$ or, alternatively, as arising from an effective epsilon-near-zero (ENZ) effect ${ }^{35-37}$. We couple this nano-coax ENZ mode to the lattice vibrations of $\mathrm{SiO}_{2}$ and demonstrate MIR USC with a mode splitting exceeding $50 \%$ of the vibrational transition frequency $(\eta>0.25)$ within a solid-state system.

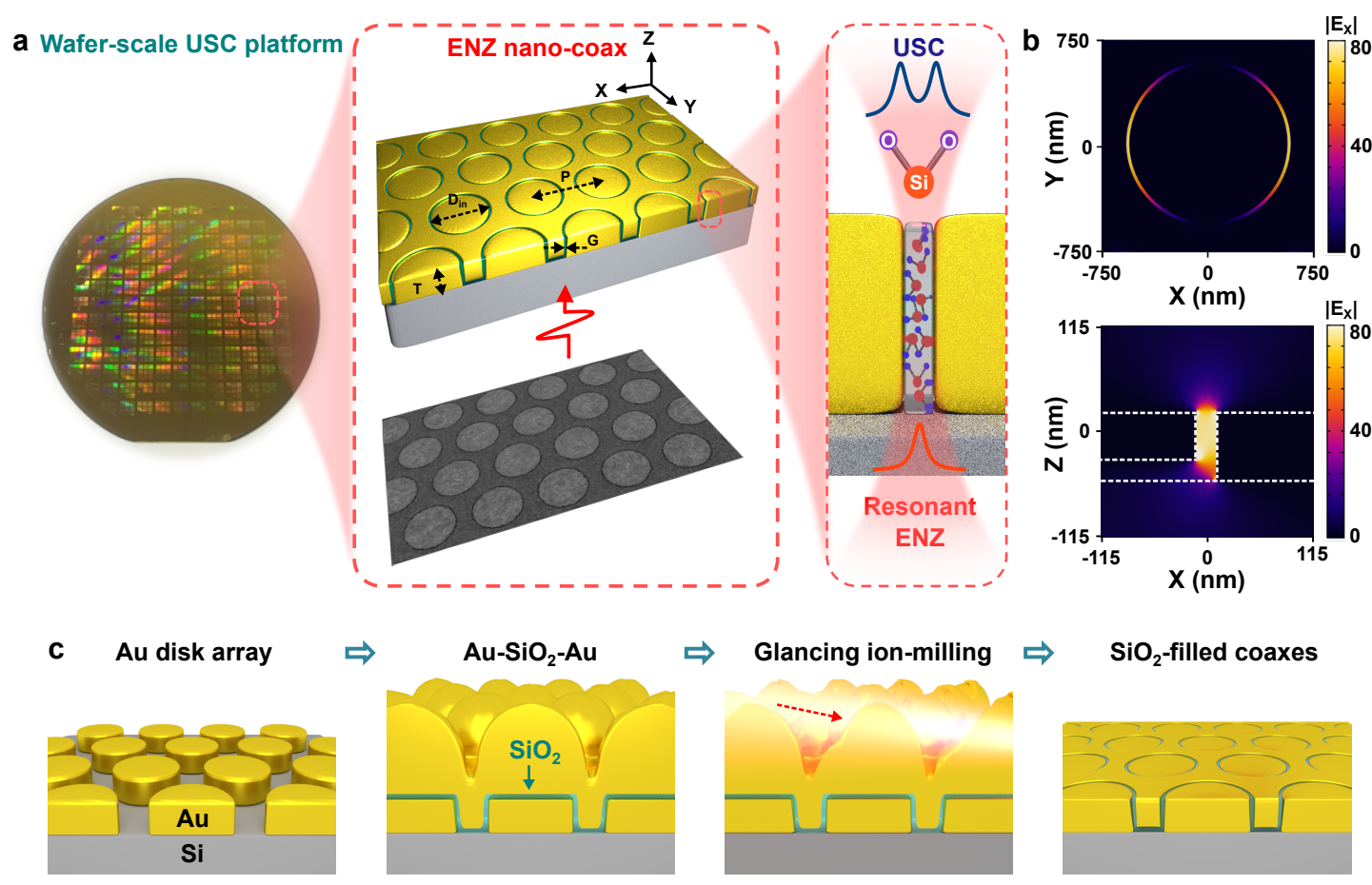

Figure 1. Wafer-scale resonant ENZ nanocavity platform for USC. (a) Geometrical parameters and illustration of $\mathrm{SiO}_{2}$ phonons interacting with the ENZ mode of the nanocavity. Inset: scanning electron micrograph of coaxial nanocavities with 21 $\mathrm{nm}$ gap, $790 \mathrm{~nm}$ diameter, $1190 \mathrm{~nm}$ period, and $80 \mathrm{~nm}$ Au thickness. (b) Computed field profiles (for fields polarized in the lateral direction) of a coaxial nanocavity (diameter $1120 \mathrm{~nm}$ and gap width $21 \mathrm{~nm}$ ) showing a lateral section (upper) and a vertical section (lower) of the ENZ transmission resonance. (c) Process flow based on ALD and wafer-scale glancing-angle ion milling. The dashed red arrow represents the direction of the glancing-angle ion beam.

\section{Results}

Design and fabrication of ENZ nanocavities. In our USC platform, $\mathrm{SiO}_{2}$-filled coaxial nanoapertures fabricated into a metal (Au) film of thickness $T$ are hexagonally arranged with varied gap size $(G)$, diameter $(D)$, and lattice periodicity $(P)$ (Fig. 1a). Unlike conventional approaches of selectively etching annular gaps in metal films, we adopt a novel fabrication approach called atomic layer lithography ${ }^{36,38}$ to create dielectric-filled nanogaps in metal films (Fig. 1c and Methods). After employing standard photolithography to define Au disk arrays on a silicon wafer, $\mathrm{SiO}_{2}$ films are conformally grown via atomic layer deposition $^{38}$ (ALD) on the exposed surfaces and sidewalls, followed by subsequent metal cladding layer deposition and planarization via glancing-angle ion milling. This batch process can produce wafer-sized arrays of coaxial apertures with a gap size down to $1 \mathrm{~nm}$, limited only by the ALD growth.

For very narrow gaps, $\mathrm{TE}_{11}$ coaxial modes have a strong plasmonic character. Such modes are characterized by an effective dielectric constant that is approximately zero at cutoff. The resonance frequency of the cavities can be shifted toward longer wavelengths without sacrificing modal confinement by increasing the coax diameter without changing the gap width. In addition, the very long wavelength associated with near-zero permittivity produces a spatially uniform optical field (Fig. 1b lower panel), which in turn provides efficient coupling to the material within the gaps.

We note that the ENZ resonance is a single-aperture effect; in other words, the existence of an array is not required for the transmission resonance we utilize (Supplementaty Section 2). We also note that the resonant optical properties of the nanocavities are robust against process variations and inhomogeneous broadening for two reasons. First, the critical dimension 
(i.e. the width of the nanogap) is lithography-independent and precisely defined by the thickness of the $\mathrm{SiO}_{2}$ film grown by ALD. Second, the ENZ mode (i.e., the zeroth-order Fabry-Perot resonance) is independent of the cavity length, and is thus insensitive to variations in the Au film thickness.

We designed a series of cavities with diameters ranging from $430 \mathrm{~nm}$ to $1120 \mathrm{~nm}$ in $30 \mathrm{~nm}$ steps, in order to tune the bare ENZ resonance from approximately 1000 to $2500 \mathrm{~cm}^{-1}$. This range is chosen to cover the Reststrahlen band of $\mathrm{SiO}_{2}$, which is defined by the transverse optical (TO) and longitudinal optical (LO) phonon frequencies (Fig. 2a). Coaxial nanocavities at the ENZ condition show extraordinary optical transmission (EOT) ${ }^{39}$, since incident light with wavelengths 4-10 $\mu$ m can pass through annular gaps that are over thousand times narrower than the wavelength, with the absolute transmittance as high as $60 \%$.

Transmission spectra through the nanocavity arrays were measured by far-field Fourier transform infrared (FTIR) spectroscopy over a large-area $(5 \mathrm{~mm} \times 5 \mathrm{~mm})$ chip containing arrays of coaxial nanocavities. The normalized transmission spectra for coaxial nanocavities filled with $21 \mathrm{~nm}$-thick $\mathrm{SiO}_{2}$ are plotted in Fig. 2b. The observed transmission features a clear anticrossing behaviour characteristic of SC.
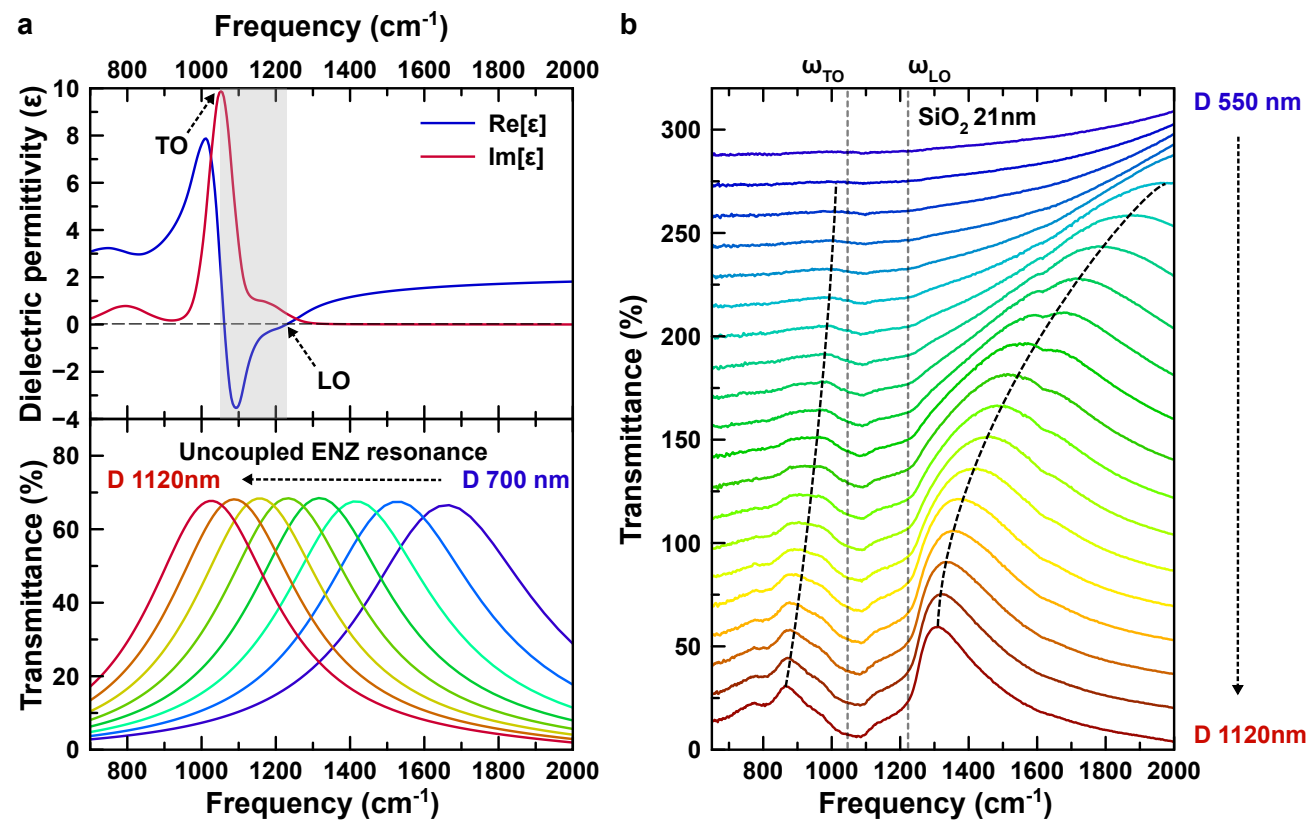

Figure 2. Normal mode splittings due to ultrastrong plasmon-phonon coupling. (a) Dielectric function ${ }^{50}$ of $\mathrm{SiO}_{2}$ (upper) and epsilon-near-zero transmission resonances (lower) of coaxial nanocavities (gap width of $21 \mathrm{~nm}$, diameter from 700 $\mathrm{nm}$ to $1120 \mathrm{~nm}$ ), simulated with a frequency-independent dielectric constant $\varepsilon_{\infty}$ inside the aperture. This calculation shows that the bare cavity resonance sweeps through the entire Reststrahlen band (gray region, upper panel) for the considered diameters (Supplementary Section 1). (b) Normalized experimental transmission spectra of $\mathrm{SiO}_{2}$-filled coaxial Au nanocavities, for gap width of $21 \mathrm{~nm}$ and diameter from 550 to $1120 \mathrm{~nm}$. Black dashed guidelines indicate the lower and upper polariton branches.

Although these results suggest that the $\mathrm{SiO}_{2}$ phonons and cavity photons are strongly coupled, the split transmission peaks could potentially be interpreted as the result of a single broad transmission resonance being quenched in a central frequency range by the strong vibrational absorption close to $\omega_{T O}$. To clarify the origin of the split transmission peaks, we perform a theoretical analysis of the coupled system to be compared to our experimental results.

\section{Theoretical analysis of ultrastrong plasmon-phonon coupling}

Classical description. Electromagnetic propagation in waveguides is usually based on a description where the phononic degrees of freedom have been integrated out, providing a frequency-dependent dielectric function $\varepsilon(\omega)$ for the propagation of the photon (Supplementary Section 3). However, in order to determine whether the system is in the strong-coupling regime, it is essential to retain both phononic and photonic degrees of freedom. We therefore consider a given waveguide mode $M$, characterized by a wavevector $k$ along the waveguide axis (in this case, $\mathrm{M}=\mathrm{TE}_{11}$, the fundamental mode of the coaxial waveguide). When the aperture is filled with a uniform dielectric constant $\varepsilon_{\infty}$ (originating from coupling to electronic degrees of freedom), the electric 
field, $\mathbf{E}(\mathbf{r})=E \mathbf{E}_{M}(\mathbf{r}, k)$, where $\mathbf{r}$ is the vector position, satisfies the wave equation

$$
\nabla \times \nabla \times E \mathbf{E}_{M}-\varepsilon_{\infty} \frac{\omega_{k}^{2}}{c^{2}} E \mathbf{E}_{M}=0,
$$

where $E$ is the field amplitude, $\mathbf{E}_{M}$ is a normalized transverse solution of Maxwell's equations (Supplementary Section S4), and $c$ is the vacuum speed of light in vacuum. When the phononic material fills the waveguide, we assume a local relation (valid for non-dispersive vibrational modes) between $\mathbf{E}$ and the relative displacement of ions, $\mathbf{x}$, following Ref. 40 . Then, for a given mode profile, $\mathbf{x}(\mathbf{r})=x \mathbf{E}_{M}(\mathbf{r}, k)$, with

$$
\begin{aligned}
\ddot{x} & =\gamma_{11} x+\gamma_{12} E, \\
P & =\gamma_{12} x+\gamma_{22} E,
\end{aligned}
$$

where $P$ is the amplitude of polarization vector $\mathbf{P}=P \mathbf{E}_{M}$ and $\ddot{x}$ is the second derivative of $x$ with respect to time. The phenomenological parameters $\gamma_{i j}$ can be written in terms of measurable quantities: $\gamma_{12}^{2}=\omega_{p}^{2} \varepsilon_{\infty} / 4 \pi$ with $\omega_{p}^{2}=\omega_{L O}^{2}-\omega_{T O}^{2}$, $\gamma_{11}=-\omega_{T O}^{2}$, and $\gamma_{22}=\left(\varepsilon_{\infty}-1\right) / 4 \pi$ (Supplementary Section 3). Note that $\gamma_{22}$ incorporates the effect of the electronic resonances excited at higher frequencies. The wave equation becomes, in this case,

$$
\nabla \times \nabla \times E \mathbf{E}_{M}-\frac{\omega^{2}}{c^{2}}(E+4 \pi P) \mathbf{E}_{M}=0 .
$$

By inspection, we find that $\mathbf{E}_{M}(\mathbf{r}, k)$ satisfies Eq. (4) if $\omega^{2}(E+4 \pi P)=\varepsilon_{\infty} \omega_{k}^{2} E$ (strictly speaking, we are neglecting the variation between $\omega$ and $\omega_{k}$ in the impedance of the metal surrounding the waveguide; for a deeper analysis of how this affects the above condition, see Supplementary Sections 2 and 5). This condition, together with Eqs. (2) and (3), can be expressed in a matrix form, stating that $\mathbf{E}_{M}(\mathbf{r}, k)$ is still a solution of Maxwell equations but at a frequency $\omega$ satisfying

$$
\left(\begin{array}{cc}
\omega^{2}-\omega_{T O}^{2} & \omega \omega_{p} \\
\omega \omega_{p} & \omega^{2}-\omega_{k}^{2}
\end{array}\right)\left(\begin{array}{c}
\omega x \\
\sqrt{\varepsilon_{\infty} / 4 \pi} E
\end{array}\right)=0
$$

(See Supplementary Section 6 for a detailed derivation and a discussion of the effects of losses) Note that the simpler coupledharmonic-oscillator model that is commonly used for fitting experimental data (Supplementary Section 7) does not show the $\omega$-dependence of the off-diagonal terms and therefore cannot describe the polaritonic branches correctly (Fig. 3a and Supplementary Fig. 6).

Quantum-mechanical description. We have used a classical approach above. For future work, especially the investigation of nonlinear optical effects, a full quantum mechanical description will be required, wherein off-resonant terms may be important and the rotating-wave approximation cannot necessarily be applied ${ }^{41-45}$. We therefore apply the canonical procedure of second quantization to the classical Hamiltonian (see Supplementary Section 9 for details). For the sake of convenience, absorption is neglected and the walls of the coaxial cavity are assumed to be perfectly conducting. We obtain a Hopfield-like Hamiltonian ${ }^{41}$ for interacting photons and phonons: $H=H_{\text {photon }}+H_{\text {phonon }}+H_{\text {int }}$, with

$$
\begin{aligned}
H_{\text {photon }}= & \sum_{m} \hbar \omega_{m}\left(a_{m}^{+} a_{m}+\frac{1}{2}\right), \\
H_{\text {phonon }}= & \sum_{m} \hbar \omega_{T O}\left(b_{m}^{+} b_{m}+\frac{1}{2}\right), \\
H_{\text {int }}= & \sum_{m} \hbar\left[i C_{m}\left(a_{m}^{+} b_{m}-a_{m} b_{m}^{+}\right)+D_{m}\left(2 a_{k}^{+} a_{m}+1\right)\right. \\
& \left.+i C_{m}\left(a_{m} b_{m}-a_{m}^{+} b_{m}^{+}\right)+D_{m}\left(a_{m} a_{m}+a_{m}^{+} a_{m}^{+}\right)\right] .
\end{aligned}
$$

where $a^{+}(a)$ and $b^{+}(b)$ are the creation (annihilation) operators for photons and phonons, respectively, the sum is over modes $m$, and

$$
C_{m}=\frac{\omega_{p}}{2} \sqrt{\frac{\omega_{T O}}{\omega_{m}}}, D_{m}=\frac{\omega_{p}^{2}}{4 \omega_{m}} .
$$

As expected, given that the system comprises two coupled harmonic oscillators, diagonalizing the full Hamiltonian gives eigenfrequencies identical to those obtained from Eq. 5. In other words, the classical approach can be used to compute the polaritonic branches exactly when the system enters into the USC regime. The same behaviour is valid for an arbitrary number of vibrational modes in the polar material (Supplementary Sections 8 and 10). 
Experimental verification of dispersion and USC. We can therefore analyse the dependence of the ENZ resonant frequencies on the coax inner diameter using Eq. 5 (Fig. 3 c-f). As $\omega_{p}$ is independent of the hole shape and size, the ENZ resonances in this strong-coupling condition depend only on the bare ENZ frequency, $\omega_{E N Z}(D)$. This bare ENZ frequency, in turn, is obtained from the upper polariton branch (see Sec. S12). This procedure is followed because the lower polariton is more affected by additional vibrational resonances that have not been considered in this simple analysis.

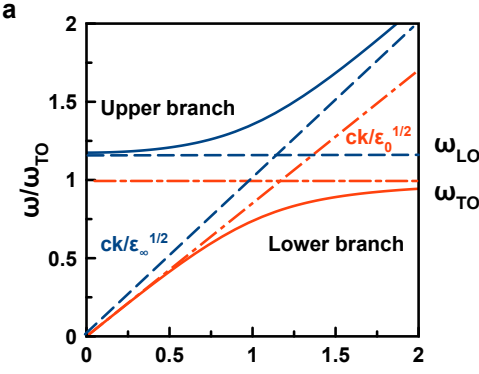

c

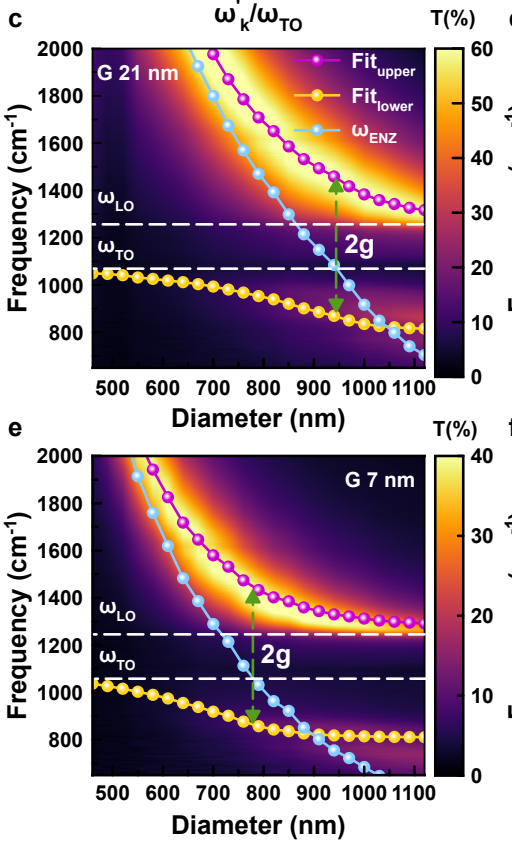

b

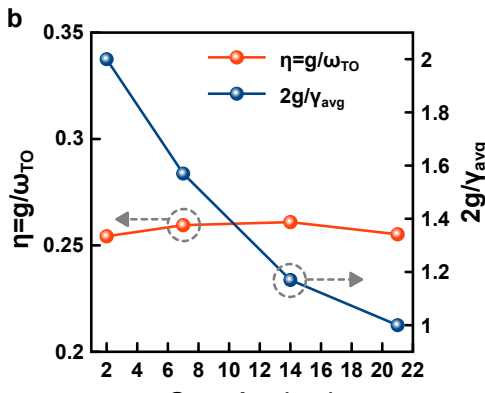

d

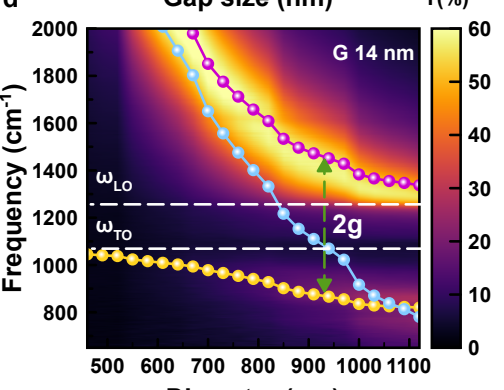

g

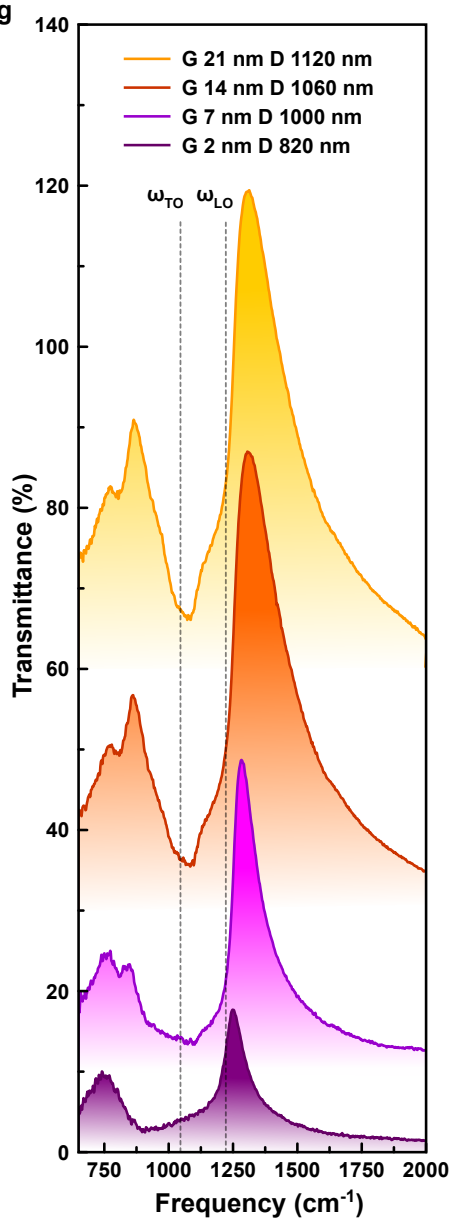

Figure 3. Dispersion mapping and validation of theoretical model. (a) The dispersion relation of bulk phonon polaritons supported in $\mathrm{SiO}_{2}$ as calculated by Eq. 5 under the assumption that a single vibrational mode is excited. (b) The measured normalized coupling strength, $\eta$, and normalized mode splitting, $\left(2 g / \gamma_{a v g}\right)$, as a function of gap size. (c-f) Dispersion maps of experimentally measured transmission $(T)$ spectra for coaxial apertures filled with $\mathrm{SiO}_{2}$ with gap thicknesses of $21 \mathrm{~nm}(\mathrm{c}), 14$ $\mathrm{nm}$ (d), $7 \mathrm{~nm}$ (e), and $2 \mathrm{~nm}$ (f). Polaritonic resonances analytically fit using Eq. S14 (purple (upper) and yellow (lower) circle dots and solid line). $\omega_{E N Z}$ (blue circles with a solid line) is used as a fitting parameter and indicates the resonance of the ENZ mode uncoupled from the polar phonons of $\mathrm{SiO}_{2}$. The coupling strength, $g$, is measured at the intersection between the uncoupled ENZ resonance and $\omega_{T O}$ (when the detuning is zero). (g) Transmission spectra measured when the bare ENZ mode crosses through the Reststrahlen band, showing distinct modal splitting and sharp resonance peaks (60\% transmittance for the $21 \mathrm{~nm}$-gap coax). Curves are shifted along the vertical axis for clarity.

For each gap size, we estimate the coupling strength $g$ as half the frequency difference between the upper and lower polariton branches when $\omega_{E N Z}(D)=\omega_{T o}$. Results are summarized in Figure $3 \mathrm{~b}$ and Table 1. Since the $\mathrm{SiO}_{2}$ layer is added to our coaxial nanocavity during the fabrication process, it is not trivial to experimentally measure the bare ENZ resonance (i.e., to measure the transmission of the cavity with an empty gap). Instead, we measure the linewidth of a bare ENZ resonance at a higher frequency, where it is uncoupled from the $\mathrm{SiO}_{2}$ phonons. As shown in Table 1, all cavities exhibit modal splittings that are larger than the average of the linewidths of the bare cavity resonances and of the TO phonon $\left(2 g / \gamma_{\text {avg }}>1\right)$. This indicates 
that the system is in the strong-coupling regime.

Moreover, the normalized coupling strength $\eta=g / \omega_{T O}>0.25$, regardless of gap width, indicating that the systems are also in the USC regime ${ }^{19}$. For a single dipole emitter in a cavity of volume $V, g \propto 1 / \sqrt{V}$. However, for collective coupling, as in our system, $g \propto \sqrt{N / V}$, where $N$ is the number of dipoles coherently coupled to the cavity mode. Thus, $g$ depends only on the density of molecules, $N / V$, and is independent of the coaxial gap width (Supplementary Section 3).

\begin{tabular}{ccccc}
\hline \hline $\mathrm{SiO}_{2}$ gap width $(\mathrm{nm})$ & 2 & 7 & 14 & 21 \\
\hline Bare ENZ mode linewidth $(\mathrm{FWHM})\left(\mathrm{cm}^{-1}\right)$ & 453 & 619 & 844 & 990 \\
Average of bare ENZ and TO phonon linewidths, $\gamma_{\text {avg }}\left(\mathrm{cm}^{-1}\right)$ & 265 & 348 & 481 & 534 \\
Normal mode splitting, $2 \mathrm{~g}\left(\mathrm{~cm}^{-1}\right)$ & 534 & 546 & 549 & 537 \\
Normalized mode splitting, $2 \mathrm{~g} / \gamma_{\text {avg }}$ & 2.02 & 1.57 & 1.14 & 1.01 \\
Normalized coupling strength, $\eta=g / \omega_{T O}$ & 0.254 & 0.26 & 0.261 & 0.255 \\
\hline FWHM, full-width at half-maximum. & & & &
\end{tabular}

Table 1. Mode splitting and normalized coupling strength.

\section{Conclusions}

We have constructed wafer-scale resonant coaxial nanocavities and achieved USC between the ENZ mode of the cavities and $\mathrm{SiO}_{2}$ phonons with a normalized coupling strength greater than 0.25 . The large magnitude of the splitting we observe - wider than the Reststrahlen band - shows that the coupling strength is truly within the USC regime, and is not the manifestation of absorption induced within the forbidden band. To further elucidate this point, we also presented a theoretical framework (both classical and quantum mechanical) that quantitatively explains the data, and which can be applied to similar USC involving homogeneous materials, waveguides, and resonant cavities.

The ENZ nanocavities can be filled with a variety of vibrationally-active materials, thus enabling USC to be realized throughout the MIR, far-IR, and terahertz spectral ranges. Furthermore, since single-nanometre-thick phononic films separate the core and cladding electrodes in each coax, the platform provides a route to combine electron tunnelling with vibrational USC and EOT, which should enable observation of ground-state electroluminescence ${ }^{46}$ and the dynamic Casimir effect ${ }^{22,23}$. The ability to reach the USC regime in mass-produced ENZ cavities with ultrasmall gap volumes $\left(\approx \lambda^{3} / 10^{7}\right)$ can also open up new avenues to explore quantum nonlinear optical processes ${ }^{47}$, multiphoton effects, higher-order nonlinear effects, and single-photon excitation of multiple atoms ${ }^{48}$, which may lead to novel applications in quantum sensing, spectroscopy, optoelectronic devices, and frequency conversion ${ }^{49}$.

\section{Methods}

\section{Device fabrication}

After pre-cleaning and pre-baking steps $\left(200{ }^{\circ} \mathrm{C}\right.$ for $\left.3 \mathrm{~min}\right)$, an undoped, double-side-polished 4-inch (100) Si wafer (University wafer) is spin-coated with AZ MIR701 photoresist for 45 s at 5,000 r.p.m., followed by baking at $90{ }^{\circ} \mathrm{C}$ for $90 \mathrm{~s}$. The patterns of hexagonal hole arrays with 24 different diameters from $430 \mathrm{~nm}$ to $1120 \mathrm{~nm}$ are transferred onto the Si wafer by photolithography (Canon $2500 \mathrm{i} 3 \mathrm{stepper)} \mathrm{with} \mathrm{a} \mathrm{dose} \mathrm{of} 150 \mathrm{~mJ} / \mathrm{cm}^{2}$, followed by post-exposure bake at $110{ }^{\circ} \mathrm{C}$ and development with $\mathrm{AZ} 3000$ MIF for $60 \mathrm{~s}$. Hexagonal Au disk arrays are created on the Si wafer via directional evaporation of 3-nm Ti and 150-nm Au films (CHA, SEC 600), followed by liftoff using AZ Kwik Strip. After oxygen plasma cleaning (STS, $320 \mathrm{PC})$ at $100 \mathrm{~W}$ for 30 $\mathrm{s}$ to remove photoresist residue, the Au disk arrays are coated conformally with a thin $\mathrm{SiO}_{2}$ film using ALD (Cambridge Nano Tech Inc., Savannah) at a typical deposition rate of $1.2 \AA$ /cycle. ALD was performed using ozone precursor and water vapor at $180{ }^{\circ} \mathrm{C}$. After conformal sputtering of $3 \mathrm{~nm}$ Ti and $400 \mathrm{~nm} \mathrm{Au}$ (AJA, ATC 2200), the top surface of $400 \mathrm{~nm}$-thick Au-deposited disk patterns is planarized by glancing-angle ion milling using a $240 \mathrm{~mA} \mathrm{Ar}{ }^{+}$ion beam incident at $5^{\circ}$ from the horizontal plane (Intlvac, Nanoquest) until the top entrance of the coaxial nanocavities is exposed.

Data Availability: The data that support the plots within this paper and other findings of this study are available from the corresponding author upon reasonable request. 


\section{References}

1. Törmä, P. \& Barnes, W. L. Strong coupling between surface plasmon polaritons and emitters: a review. Rep. Prog. Phys. 78, 013901, DOI: 10.1088/0034-4885/78/1/013901 (2015).

2. Ebbesen, T. W. Hybrid light-matter states in a molecular and material science perspective. Acc. Chem. Res. 49, 2403-2412, DOI: 10.1021/acs.accounts.6b00295 (2016).

3. Pelton, M. Modified spontaneous emission in nanophotonic structures. Nat. Photonics 9, 427-435, DOI: 10.1038/nphoton. 2015.103 (2015).

4. Luk'yanchuk, B. et al. The Fano resonance in plasmonic nanostructures and metamaterials. Nat. Mater. 9, 707-715, DOI: 10.1038/nmat2810 (2010).

5. Neubrech, F. et al. Resonant plasmonic and vibrational coupling in a tailored nanoantenna for infrared detection. Phys. Rev. Lett. 101, 157403, DOI: 10.1103/PhysRevLett.101.157403 (2008).

6. Yoshie, T. et al. Vacuum Rabi splitting with a single quantum dot in a photonic crystal nanocavity. Nature 432, 200-203, DOI: 10.1038/nature03119 (2004).

7. Reithmaier, J. P. et al. Strong coupling in a single quantum dot-semiconductor microcavity system. Nature 432, 197-200, DOI: 10.1038/nature02969 (2004).

8. Aoki, T. et al. Observation of strong coupling between one atom and a monolithic microresonator. Nature 443, 671-674, DOI: 10.1038/nature05147 (2006).

9. Liu, X. et al. Strong light-matter coupling in two-dimensional atomic crystals. Nat. Photonics 9, 30-34, DOI: 10.1038/ nphoton.2014.304 (2015).

10. Benz, F. et al. Single-molecule optomechanics in "picocavities". Science 354, 726-729, DOI: 10.1126/science.aah5243 (2016).

11. Santhosh, K., Bitton, O., Chuntonov, L. \& Haran, G. Vacuum Rabi splitting in a plasmonic cavity at the single quantum emitter limit. Nat. Commun. 7, 11823, DOI: 10.1038/ncomms11823 (2016).

12. Runnerstrom, E. L. et al. Polaritonic hybrid-epsilon-near-zero modes: Beating the plasmonic confinement vs propagationlength trade-off with doped cadmium oxide bilayers. Nano Lett. 19, 948-957, DOI: 10.1021/acs.nanolett.8b04182 (2018).

13. Leng, H., Szychowski, B., Daniel, M.-C. \& Pelton, M. Strong coupling and induced transparency at room temperature with single quantum dots and gap plasmons. Nat. Commun. 9, 4012, DOI: 10.1038/s41467-018-06450-4 (2018).

14. Park, K.-D. et al. Tip-enhanced strong coupling spectroscopy, imaging, and control of a single quantum emitter. Sci. Adv. 5, eaav5931, DOI: 10.1126/sciadv.aav5931 (2019).

15. Schoelkopf, R. J. \& Girvin, S. M. Wiring up quantum systems. Nature 451, 664-669, DOI: doi.org/10.1038/451664a (2008).

16. Dunkelberger, A., Spann, B., Fears, K., Simpkins, B. \& Owrutsky, J. Modified relaxation dynamics and coherent energy exchange in coupled vibration-cavity polaritons. Nat. Commun. 7, 13504, DOI: 10.1038/ncomms13504 (2016).

17. Munkhbat, B., Wersäll, M., Baranov, D. G., Antosiewicz, T. J. \& Shegai, T. Suppression of photo-oxidation of organic chromophores by strong coupling to plasmonic nanoantennas. Sci. Adv. 4, eaas9552, DOI: 10.1126/sciadv.aas9552 (2018).

18. Thomas, A. et al. Tilting a ground-state reactivity landscape by vibrational strong coupling. Science 363, 615-619, DOI: 10.1126/science.aau7742 (2019).

19. Kockum, A. F., Miranowicz, A., Liberato, S. D., Savasta, S. \& Nori, F. Ultrastrong coupling between light and matter. Nat. Rev. Phys. 1, 19-40, DOI: 10.1038/s42254-018-0006-2 (2019).

20. Forn-Díaz, P., Lamata, L., Rico, E., Kono, J. \& Solano, E. Ultrastrong coupling regimes of light-matter interaction. Rev. Mod. Phys. 91, 025005, DOI: 10.1103/RevModPhys.91.025005 (2019).

21. Romero, G., Ballester, D., Wang, Y. M., Scarani, V. \& Solano, E. Ultrafast quantum gates in circuit QED. Phys. Rev. Lett. 108, 120501, DOI: 10.1103/PhysRevLett.108.120501 (2012).

22. Johansson, J. R., Johansson, G., Wilson, C. M. \& Nori, F. Dynamical Casimir effect in a superconducting coplanar waveguide. Phys. Rev. Lett. 103, 147003, DOI: 10.1103/PhysRevLett.103.147003 (2009).

23. Wilson, C. M. et al. Observation of the dynamical Casimir effect in a superconducting circuit. Nature 479, 376-379, DOI: 10.1038/nature10561 (2011). 
24. Schwartz, T., Hutchison, J. A., Genet, C. \& Ebbesen, T. W. Reversible switching of ultrastrong light-molecule coupling. Phys. Rev. Lett. 106, 196405, DOI: 10.1103/PhysRevLett.106.196405 (2011).

25. Niemczyk, T. et al. Circuit quantum electrodynamics in the ultrastrong-coupling regime. Nat. Phys. 6, 772-776, DOI: 10.1038/nphys1730 (2010).

26. Jouy, P. et al. Transition from strong to ultrastrong coupling regime in mid-infrared metal-dielectric-metal cavities. Appl. Phys. Lett. 98, 231114, DOI: 10.1063/1.3598432 (2011).

27. George, J. et al. Multiple Rabi splittings under ultrastrong vibrational coupling. Phys. Rev. Lett. 117, 153601, DOI: 10.1103/PhysRevLett.117.153601 (2016).

28. Scalari, G. et al. Ultrastrong coupling of the cyclotron transition of a $2 \mathrm{D}$ electron gas to a THz metamaterial. Science 335, 1323-1326, DOI: 10.1126/science.1216022 (2012).

29. Shelton, D. J. et al. Strong coupling between nanoscale metamaterials and phonons. Nano Lett. 11, 2104-2108, DOI: $10.1021 / \mathrm{nl} 200689 \mathrm{z}(2011)$.

30. Autore, M. et al. Boron nitride nanoresonators for phonon-enhanced molecular vibrational spectroscopy at the strong coupling limit. Light. Sci. Appl. 7, 17172, DOI: 10.1038/1sa.2017.172 (2018).

31. Lather, J., Bhatt, P., Thomas, A., Ebbesen, T. W. \& George, J. Cavity catalysis by cooperative vibrational strong coupling of reactant and solvent molecules. Angew. Chem. Int. Ed. 58, 10635-10638, DOI: 10.1002/anie.201905407 (2019).

32. Jin, X. et al. Reshaping the phonon energy landscape of nanocrystals inside a terahertz plasmonic nanocavity. Nat. Commun. 9, 763, DOI: 10.1038/s41467-018-03120-3 (2018).

33. Muller, E. A. et al. Nanoimaging and control of molecular vibrations through electromagnetically induced scattering reaching the strong coupling regime. ACS Photonics 5, 3594-3600, DOI: 10.1021/acsphotonics.8b00425 (2018).

34. Baida, F. I., Belkhir, A., Labeke, D. V. \& Lamrous, O. Subwavelength metallic coaxial waveguides in the optical range: Role of the plasmonic modes. Phys. Rev. B 74, 205419, DOI: 10.1103/PhysRevB.74.205419 (2006).

35. Alù, A. \& Engheta, N. Light squeezing through arbitrarily shaped plasmonic channels and sharp bends. Phys. Rev. B 78, 035440, DOI: 10.1103/PhysRevB.78.035440 (2008).

36. Yoo, D. et al. High-throughput fabrication of resonant metamaterials with ultrasmall coaxial apertures via atomic layer lithography. Nano Lett 16, 2040-2046, DOI: 10.1021/acs.nanolett.6b00024 (2016).

37. Liberal, I. \& Engheta, N. Near-zero refractive index photonics. Nat. Photonics 11, 149-158, DOI: 10.1038/nphoton.2017.13 (2017).

38. Lindquist, N. C., Nagpal, P., McPeak, K. M., Norris, D. J. \& Oh, S.-H. Engineering metallic nanostructures for plasmonics and nanophotonics. Rep. Prog. Phys. 75, 036501, DOI: 10.1088/0034-4885/75/3/036501 (2012).

39. Garcia-Vidal, F. J., Martin-Moreno, L., Ebbesen, T. W. \& Kuipers, L. Light passing through subwavelength apertures. Rev. Mod. Phys. 82, 729-787, DOI: 10.1103/RevModPhys.82.729 (2010).

40. Born, M. \& Huang, K. Dynamical theory of crystal lattices (Oxford University Press, 1988).

41. Hopfield, J. J. Theory of the contribution of excitons to the complex dielectric constant of crystals. Phys. Rev. 112, 1555-1567, DOI: 10.1103/PhysRev.112.1555 (1958).

42. Quattropani, A., Andreani, L. C. \& Bassani, F. Quantum theory of polaritons with spatial dispersion: Exact solutions. Il Nuovo Cimento D 7, 55-69, DOI: 10.1007/BF02452395 (1986).

43. Ciuti, C., Bastard, G. \& Carusotto, I. Quantum vacuum properties of the intersubband cavity polariton field. Phys. Rev. B 72, 115303, DOI: 10.1103/PhysRevB.72.115303 (2005).

44. Todorov, Y. \& Sirtori, C. Intersubband polaritons in the electrical dipole gauge. Phys. Rev. B 85, 045304, DOI: 10.1103/PhysRevB.85.045304 (2012).

45. Kéna-Cohen, S., Maier, S. A. \& Bradley, D. D. C. Ultrastrongly coupled exciton-polaritons in metal-clad organic semiconductor microcavities. Adv. Opt. Mater. 1, 827-833, DOI: 10.1002/adom.201300256 (2013).

46. Cirio, M., De Liberato, S., Lambert, N. \& Nori, F. Ground state electroluminescence. Phys. Rev. Lett. 116, 113601, DOI: 10.1103/PhysRevLett.116.113601 (2016).

47. Kockum, A. F., Miranowicz, A., Macrì, V., Savasta, S. \& Nori, F. Deterministic quantum nonlinear optics with single atoms and virtual photons. Phys. Rev. A 95, 063849, DOI: 10.1103/PhysRevA.95.063849 (2017). 
48. Garziano, L. et al. One photon can simultaneously excite two or more atoms. Phys. Rev. Lett. 117, 043601, DOI: 10.1103/PhysRevLett.117.043601 (2016).

49. Kockum, A. F., Macrì, V., Garziano, L., Savasta, S. \& Nori, F. Frequency conversion in ultrastrong cavity QED. Sci. Rep. 7, 5313, DOI: 10.1038/s41598-017-04225-3 (2017).

50. Kischkat, J. et al. Mid-infrared optical properties of thin films of aluminum oxide, titanium dioxide, silicon dioxide, aluminum nitride, and silicon nitride. Appl. Opt. 51, 6789-6898, DOI: 10.1364/AO.51.006789 (2012).

\section{Acknowledgements}

The authors thank Thomas Ebbesen for helpful comments. This research was supported by grants from the U.S. National Science Foundation (ECCS 1809240 to D.Y., D.A.M., S.-H.O.; ECCS 1809723 to I.H.L., S.-H.O.) and the Samsung Global Research Outreach (GRO) Program (to S.-H.O.). F.dL.P. and L.M.M. acknowledge financial support from the Spanish Ministry of Economy and Competitivity through projects MAT2017-88358-C3-1-R and MAT2017-88358-C3-2-R and the Aragón Government project Q-MAD. M.P. acknowledges support from the U.S. National Science Foundation (NSF DMR-1905135). M.B.R. acknowledges funding from the U.S. National Science Foundation (NSF CHE-1709822). J.D.C. was supported by the Office of Naval Research Grant N00014-18-12107. S.-H.O. further acknowledges support from the Sanford P. Bordeau Chair in Electrical Engineering at the University of Minnesota.

\section{Author contributions}

D.Y. and S.-H.O. conceived the project and designed experiments. D.Y. performed device design, fabrication, and measurements. F.dL.P. and L.M.M. developed theories and performed numerical calculations. D.A.M. and I.-H.L. performed computer simulations. D.Y., F.dL.P., M.P., D.A.M., I.-H.L., M.B.R., J.D.C., L.M.M., and S.-H.O. analysed the results. All authors contributed to interpretation of the results and wrote the paper together.

\section{Competing interests}

The authors declare no competing interests.

\section{Additional information}

Supplementary information is available for this paper.

Correspondence and requests for materials should be addressed to S.-H.O. or L.M.M. 


\title{
Ultrastrong plasmon-phonon coupling via epsilon-near-zero nanocavities
}

\author{
Daehan Yoo $^{1 \dagger}$, Fernando de León-Pérez ${ }^{2,3 \dagger}$, Matthew Pelton ${ }^{4}$, \\ In-Ho Lee ${ }^{1}$, Daniel A. Mohr ${ }^{1}$, Markus B. Raschke ${ }^{5}$, Joshua D. Caldwell ${ }^{6}$, \\ Luis Martín-Moreno ${ }^{3 *}$, and Sang-Hyun $\mathrm{Oh}^{1 *}$ \\ ${ }^{1}$ Department of Electrical and Computer Engineering, University of Minnesota, Minneapolis, MN, USA, \\ ${ }^{2}$ Centro Universitario de la Defensa de Zaragoza, Zaragoza, Spain, \\ ${ }^{3}$ Instituto de Nanociencia y Materiales de Aragón (INMA) and Departamento de Física de la Materia Condensada, \\ CSIC-Universidad de Zaragoza, Zaragoza, Spain, \\ ${ }^{4}$ Department of Physics, University of Maryland, Baltimore County, Baltimore, MD, USA, \\ ${ }^{5}$ Department of Physics and JILA, University of Colorado, Boulder, CO, USA, \\ ${ }^{6}$ Department of Mechanical Engineering, Vanderbilt University, Nashville, TN, USA.
}

*E-mail: 1mm@unizar.es and sang@umn.edu

$\dagger$ These authors contributed equally.

\section{S1 Comparison of simulations and experiments}

To further investigate the coupling between epsilon-near-zero (ENZ) and $\mathrm{SiO}_{2}$ phonon modes, we compare finite-element method (FEM) numerical modelling (dispersion map) and experimental results (white dots) in Fig. S1. The FEM-modelled dispersion agrees well with the measured data, and we observe a splitting on resonance that is about two times wider than the Reststrahlen band.

We plot in Figs. S2 and S3 the transmission spectra used for measuring the linewidth of bare and dressed ENZ resonances, respectively. Linewidths and quality factors, $Q$, for both bare and dressed modes and mode splitting of dressed modes, extracted from Figs. S2 and S3, are summarized in Table S1. Interestingly, linewidth narrowing of the coupled modes is observed in the $\mathrm{SiO}_{2}$-filled coaxial cavities, compared to the bare cavity resonances. The hybridized modes exhibit plasmon- and phonon-like properties simultaneously, resulting in linewidths that are the average of the plasmon and phonon modes. While uncoupled ENZ modes usually exhibit a $Q$-factor of $2 \sim 3$, the hybrid modes generated in the vicinity of the asymptotic limits of the LO phonon exhibits $Q>5$ for $2 \mathrm{~nm}$-gap coaxial cavities, where the coupling strength is maximum. 


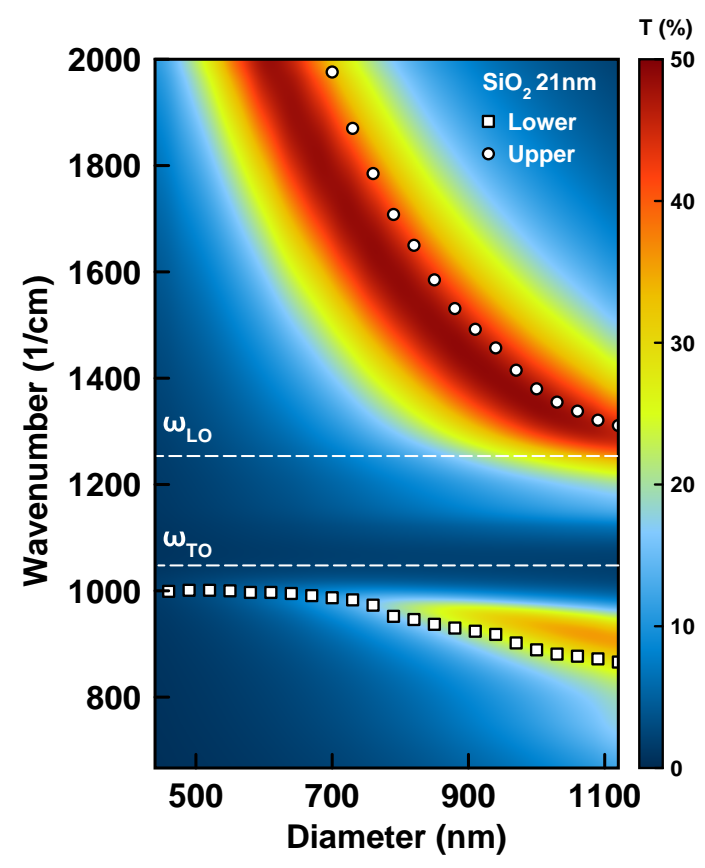

Figure S1. Colour map shows simulated transmission spectra of coaxial nanocavities ( $21 \mathrm{~nm} \mathrm{SiO} \mathrm{Sgap}_{2}$ in a gold film as a function of the coaxial diameter. White circular (square) dots indicate the upper (lower) branch spectral peak positions measured from FTIR spectra in Fig. 2 b.
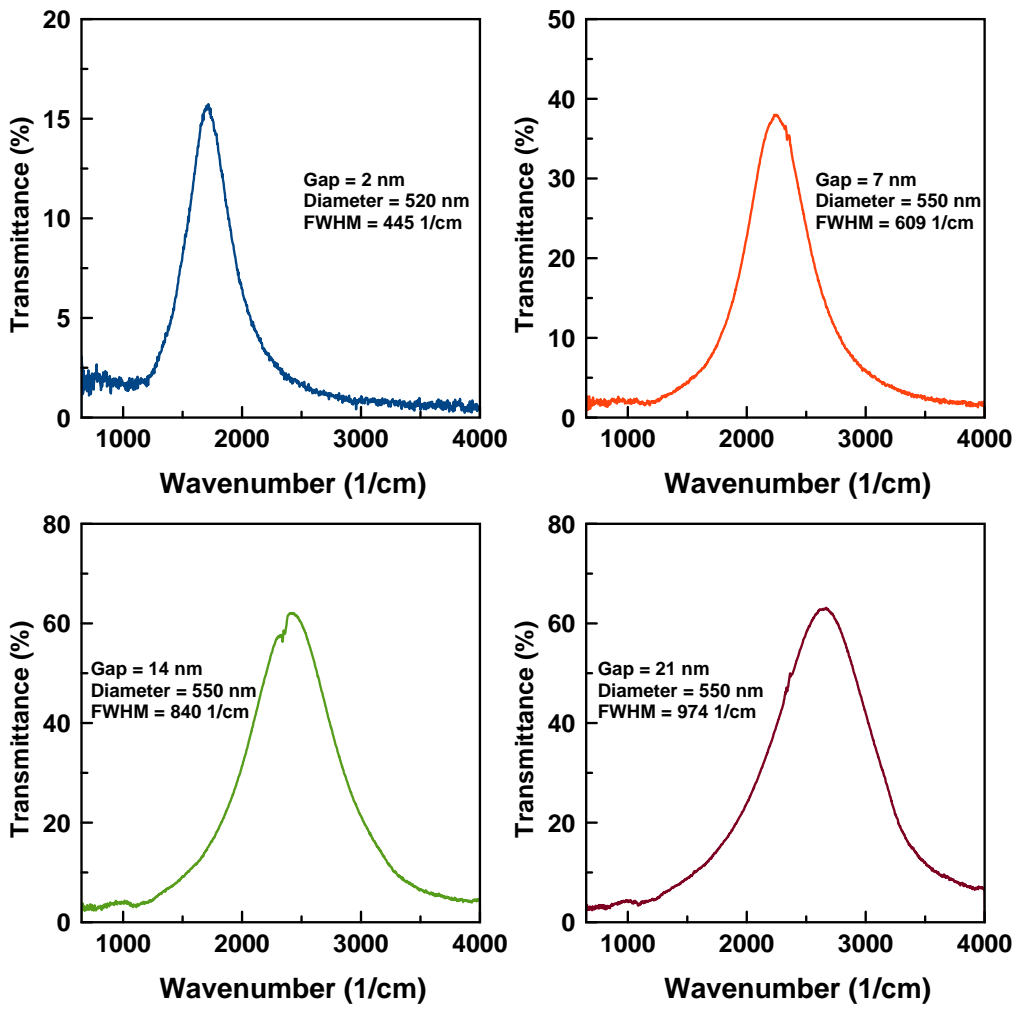

Figure S2. Transmission spectra used for measuring the linewidth of the bare epsilon-near-zero resonance. 

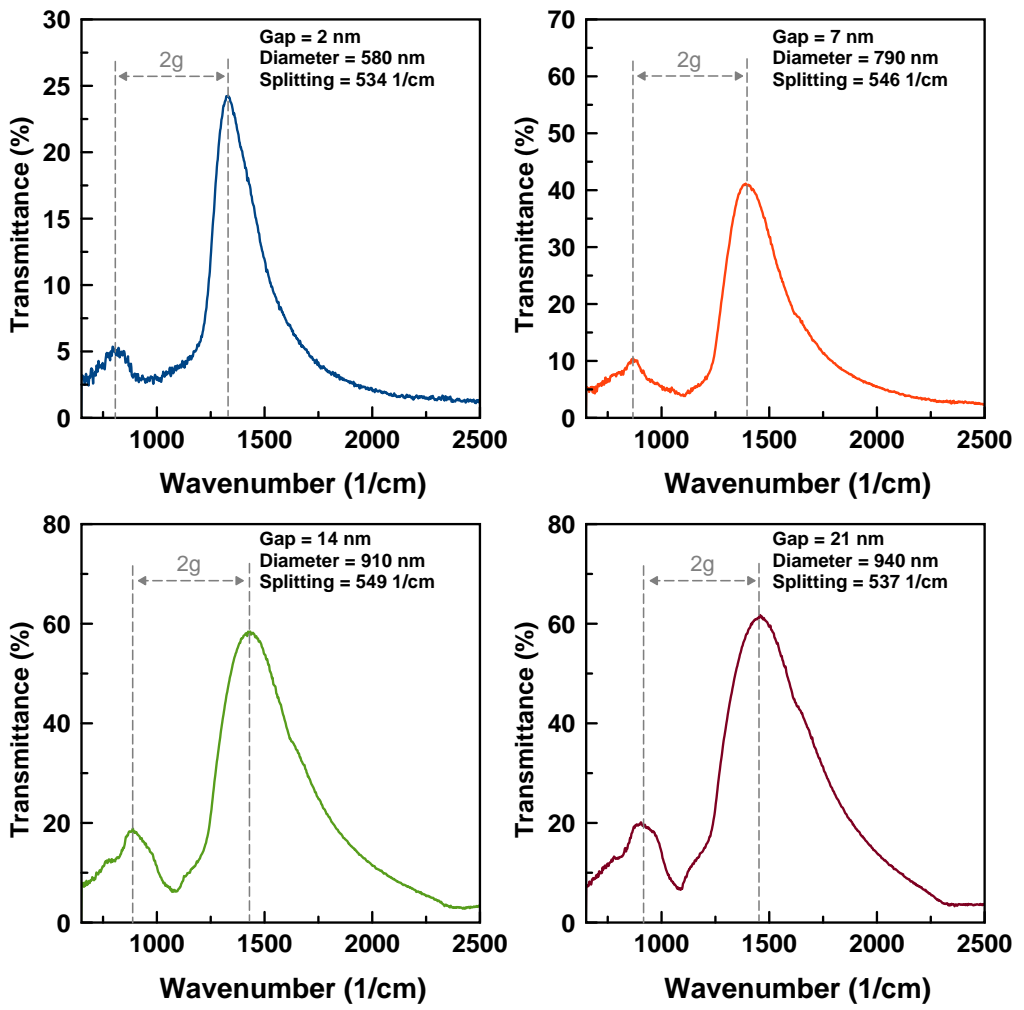

Figure S3. Transmission spectra used for measuring both the linewidth of the coupled epsilon-near-zero resonance and the resulting mode splitting.

\begin{tabular}{c|c|c|c|c|c}
\hline \hline \multicolumn{2}{|c|}{ Gap $(\mathrm{nm})$} & 2 & 7 & 14 & 21 \\
\hline \multirow{2}{*}{ Bare ENZ mode } & Diameter $(\mathrm{nm})$ & 520 & 520 & 550 & 550 \\
& Linewidth $\left(\mathrm{cm}^{-1}\right)(\mathrm{Q}$ factor $)$ & $453(3.8)$ & $619(3.6)$ & $844(2.9)$ & $990(2.7)$ \\
\hline \multirow{2}{*}{ At zero-detuned } & Diameter $(\mathrm{nm})$ & 580 & 790 & 940 & 940 \\
& Linewidth $\left(\mathrm{cm}^{-1}\right)(\mathrm{Q}$ factor) & $245(5.4)$ & $312(4.5)$ & $438(3.3)$ & $483(3.0)$ \\
\hline \multicolumn{2}{r|}{ Mode splitting $\left(\mathrm{cm}^{-1}\right)$} & 534 & 546 & 549 & 537 \\
\hline \hline
\end{tabular}

Table S1. Measured linewidths and quality factors of resonances shown in Figs. S2 and S3 for bare and dressed modes, respectively; mode splittings of dressed modes. 


\section{S2 Transmission resonances of a hole array}

Waveguide modes are the most natural choice of basis for the expansion of the electromagnetic (EM) fields inside the coaxial holes ${ }^{1}$. In numerical simulations, fast convergence is achieved with a small number of such modes. In fact, considering just the fundamental mode is an excellent approximation for deeply subwavelength holes. Reflection of these modes at the ends of the coaxial waveguide leads to Fabry-Perot (FP)-like transmission resonances. These resonances appear spectrally close to the cutoff frequency of the fundamental waveguide mode inside the hole ${ }^{1}$, when it acts as an ENZ medium. ${ }^{2}$ When holes are arranged periodically, additional transmission resonances develop at wavelengths slightly red-shifted with respect to the array periodicity $(P)$, due to the excitation of surface modes. A strong hybridization of these two kinds of transmission resonances occurs when the cutoff wavelength of a FP resonance is near $P$. However, our coaxial aperture arrays are designed such that FP transmission resonances appear in the mid-infrared (MIR) while surface resonances are in the near-infrared, thus, FP and surface modes do not hybridize.

Fig. S4 compares the transmittance of the hole array with the square of the effective index, $q_{z}^{2}=c^{2} k_{z}^{2} / \omega^{2}$. We note that $\operatorname{Im}\left[k_{z}^{2}\right]$ does not vanish at cutoff due to the absorption losses. Even for uncoupled cavity modes, when the hole is filled with a lossless medium with dielectric constant $\varepsilon_{\infty}$, losses in the metal give $\operatorname{Im}\left[k_{z}^{2}\right] \neq 0$; see the magenta line in Fig. S4.

Finally, it is worth noting that in the structures we consider, the gap sizes (between $G=2 \mathrm{~nm}$ and $G=21 \mathrm{~nm})$ are smaller than the skin depth $(\sim 30 \mathrm{~nm})$. This means the metal cannot be approximated as a perfect electrical conductor (PEC), and both the field profile and the dispersion relation strongly depend on the penetration inside the metal. This field penetration must therefore be taken into account in the calculation. This is illustrated by comparing the cutoff wavelengths for waveguides with gold or PEC sidewalls in Fig. S4(c). The result is that the fundamental waveguide mode no longer has a pure TM or TE character (as it would for a PEC), but is instead a linear combination of the two polarizations. However, for the process of transmission and the range of frequencies considered, we find that the TE component couples to the external illumination much more efficiently than the TM one, so the mode behaves effectively as a quasi-TE mode.

\section{S3 Lattice model for a homogeneous medium}

For completeness, this section summarizes the theory developed by Born and Huang ${ }^{3}$ for the optical phonons in a diatomic crystal. In this case, there are three optical phonon branches (one longitudinal and two transverse). Unit cells with a larger number of 

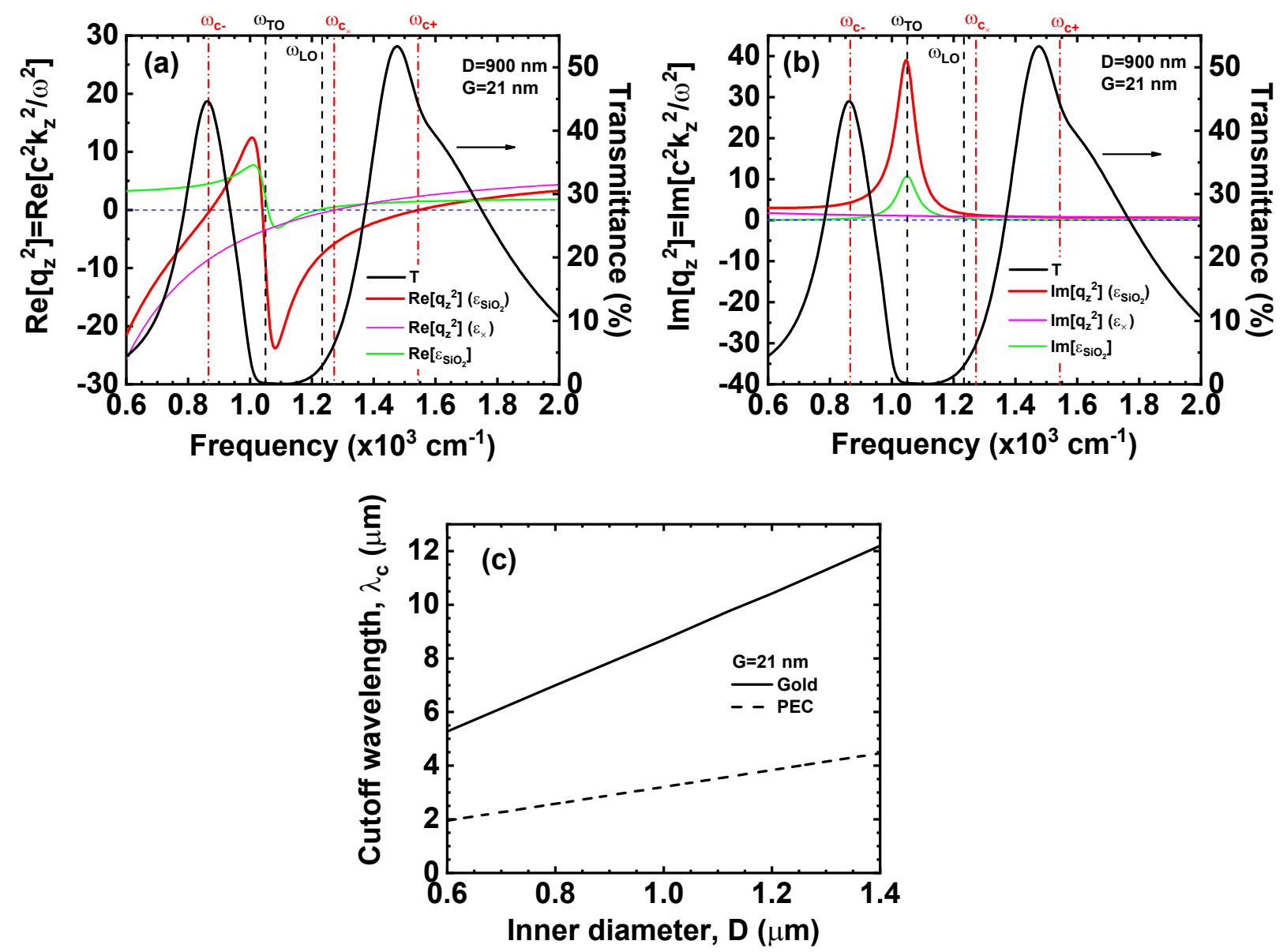

Figure S4. (a) Real and (b) imaginary parts of the square of the effective index, $q_{z}^{2}$ (red line), as a function of a frequency for the fundamental $\mathrm{TE}_{11}$ mode of an $\mathrm{SiO}_{2}$-filled coaxial waveguide with diameter $D=900 \mathrm{~nm}$ and gap size $G=21 \mathrm{~nm}$. This quantity is compared with $q_{z}^{2}$ for a $\varepsilon_{\infty}$ filled gap (magenta line) and with the dielectric constant of $\mathrm{SiO}_{2}$ (green line). The cutoff frequencies (red dash-dotted vertical lines) of the $\mathrm{SiO}_{2}$-filled gap are computed with Eq. (S9). The transmittance of a hexagonal hole array with $1190 \mathrm{~nm}$ period and $80 \mathrm{~nm}$ Au thickness (black line) shows peaks when the holes are at cutoff; i.e., for $\operatorname{Re}\left[q_{z}^{2}\right]=0$. The coupled-mode method was employed for computing the transmission spectrum ${ }^{1}$. (c) The cutoff wavelength for a waveguide with Au sidewalls, $\lambda_{c}$, is compared with the cutoff wavelength for a waveguide with perfect-electrical-conductor sidewalls, $\lambda_{c}^{P E C}=\pi(D+G)$, as a function of the inner diameter of the hole, $D$.

atoms $(s)$, and correspondingly 3(s-1) optical branches, will be discussed in Sec. S8.

For polarizable (nonrigid) ions in the diatomic cell, the equation of motion and the polarization are given by

$$
\begin{aligned}
\ddot{\mathbf{x}} & =\gamma_{11} \mathbf{x}-\gamma_{13} \dot{\mathbf{x}}+\gamma_{12} \mathbf{E}, \\
\mathbf{P} & =\gamma_{12} \mathbf{x}+\gamma_{22} \mathbf{E},
\end{aligned}
$$

where the normalized coordinate $\mathbf{x}=\left(\mathbf{u}_{+}-\mathbf{u}_{-}\right) / \sqrt{\mu n}$ depends on the relative displacement of the positive and negative ions, $\mathbf{u}_{+}-\mathbf{u}_{-}$, the number of cells per unit volume, $n$, and the reduced mass, $\mu=m_{+} m_{-} /\left(m_{+}+m_{-}\right)$. $\mathbf{P}$ and $\mathbf{E}$ are the dielectric polarization and electric field, respectively. By considering harmonic solutions, where the time dependence of all fields has the form $\mathbf{x}=\mathbf{x}_{0} \exp (-i \omega t)$, we can eliminate 
$\mathbf{x}=-\gamma_{12} \mathbf{E} /\left(\omega^{2}+\gamma_{11}+i \gamma_{13} \omega\right)$ from these equations, and relate $\mathbf{P}$ and $\mathbf{E}$ by the equation $\mathbf{P}=\left[\gamma_{22}-\gamma_{12}^{2} /\left(\omega^{2}+\gamma_{11}+i \gamma_{13} \omega\right)\right] \mathbf{E}$. From the definition of the dielectric displacement, $\mathbf{D}=\mathbf{E}+4 \pi \mathbf{P}=\varepsilon \mathbf{E}$, we obtain the dielectric function,

$$
\varepsilon(\omega)=1+4 \pi \gamma_{22}-4 \pi \gamma_{12}^{2} /\left(\omega^{2}+\gamma_{11}+i \gamma_{13} \omega\right) .
$$

If $\varepsilon(\omega)$ is written in terms of measurable quantities,

$$
\varepsilon(\omega)=\varepsilon_{\infty}\left(1+\frac{\omega_{p}^{2}}{\omega_{T O}^{2}-\omega^{2}-i \gamma \omega}\right),
$$

the $\gamma$-coefficients can be expressed as $\gamma_{22}=\left(\varepsilon_{\infty}-1\right) / 4 \pi, \gamma_{12}=\left(\varepsilon_{\infty} / 4 \pi\right)^{1 / 2} \omega_{p}, \gamma_{13}=\gamma$, and $\gamma_{11}=-\omega_{T O}^{2}$.

If we neglect the induced internal polarization in the ions (i.e., if we consider them as rigid), the vibrational coupling constant $\omega_{p}$ can be estimated to be ${ }^{3}$

$$
\omega_{p}=\sqrt{\frac{4 \pi n e^{2}}{M}}
$$

\section{S4 Wave equation}

The wave equation may be derived from Maxwell's equations (using the Gaussian convention):

$$
\begin{aligned}
\nabla \times \mathbf{H} & =\frac{1}{c} \frac{\partial \mathbf{D}}{\partial t}, \\
\nabla \times \mathbf{E} & =-\frac{1}{c} \frac{\partial \mathbf{H}}{\partial t},
\end{aligned}
$$

in the following way. After assuming harmonic solutions $(\sim \exp (-i \omega t))$, we can apply the operator $\nabla \times$ on (S5) and replace (S4) into the resulting equation to give

$$
\nabla \times \nabla \times \mathbf{E}-\frac{\omega^{2}}{c^{2}} \mathbf{D}=0
$$

Using the constitutive relation for the displacement current $\mathbf{D}=\mathbf{E}+4 \pi \mathbf{P}=\varepsilon \mathbf{E}$, we obtain Eqs.(1) and (4) of the main text.

\section{S5 Scaling relation}

At a given frequency $\omega$ and for a general dielectric constant $\varepsilon(\mathbf{r})$, the wave equation for the electric field becomes

$$
\nabla \times \nabla \times \mathbf{E}(\mathbf{r})-\varepsilon(\mathbf{r}) \frac{\omega^{2}}{c^{2}} \mathbf{E}(\mathbf{r})=0 .
$$


The field thus depends only on the combination $\kappa^{2}(\mathbf{r}) \equiv \varepsilon(\mathbf{r})(\omega / c)^{2}$. For a waveguide defined by an internal dielectric region (characterized by $\varepsilon_{d}$ ) and an external metallic region (with $\varepsilon_{m}$ ), the field depends only on the values of the corresponding $\kappa_{d}^{2}$ and $\kappa_{m}^{2}$. The scaling relation

$$
\varepsilon_{d}, \varepsilon_{m}, \omega \rightarrow \varepsilon_{d} \zeta, \varepsilon_{m} \zeta, \omega / \sqrt{\zeta}
$$

leaves $\kappa_{d}^{2}$ and $\kappa_{m}^{2}$ unchanged for any $\zeta$. However, for a waveguide made from a given metal, if the material inside the waveguide is replaced by a different dielectric, the scaling relation will not be fulfilled, as the spectral dependence of $\varepsilon_{m}$ will generally not follow the scaling imposed by the change in $\varepsilon_{d}$. Nevertheless, we find two relevant exceptions: a perfect electrical conductor and a Drude metal at low frequencies.

For a PEC, $\varepsilon_{m}=-\infty$. In that case, the dependence on $\varepsilon_{m}$ disappears from the scaling relation (as $\varepsilon_{m} \zeta=-\infty$, independent of $\zeta$ ). If Eq. (S7) holds and the waveguide is filled with a material with dielectric function $\varepsilon_{d}^{\prime}(\omega)$, the electromagnetic fields satisfy the same Maxwell's equation with $\kappa_{d}^{\prime 2}=\varepsilon_{d}^{\prime}(\omega)(\omega / c)^{2}$. The solution $\mathbf{E}$ for $\varepsilon_{d}$ at a given frequency $\omega$ is thus the same as for $\varepsilon_{d}^{\prime}\left(\omega^{\prime}\right)$ at a different $\omega^{\prime}$ given by

$$
\varepsilon_{d}^{\prime}\left(\omega^{\prime}\right) \omega^{\prime 2}=\varepsilon_{d} \omega^{2}
$$

A similar behaviour is found for a Drude metal with dielectric function $\varepsilon_{m}(\omega)=1-$ $\omega_{p m}^{2} / \omega^{2}$. At frequencies much smaller than the plasma frequency $\omega_{p m}$ (i.e., at midinfrared and smaller frequencies) $\kappa_{m}^{2} \approx-\omega_{p m}^{2} / c^{2}$ and does not depend on $\omega$. Therefore, field patterns depend only on $\omega_{p m}$ and $\kappa_{d}^{2}$. This limit holds for the spectral range covered by the experiments.

In particular, Eq. (S8) is fulfilled when the field pattern corresponds to the cutoff condition $k_{z}^{2}=0$, where $k_{z}$ is the component of the wavevector along the waveguide $(z)$ axis. Thus, if we know the cutoff frequency $\omega_{c_{\infty}}$ when a waveguide is filled with a material characterized by $\varepsilon_{\infty}$, we can obtain the cutoff frequency when the material filling the waveguide is characterized by a dielectric constant $\varepsilon(\omega)$ :

$$
\varepsilon\left(\omega_{c}\right) \omega_{c}^{2}=\varepsilon_{\infty} \omega_{c_{\infty}}^{2} .
$$

We note that, in the region of anomalous dispersion, this equation may (and in the case of SC does) have more than one solution.

Eq. (S9) remains an excellent approximation even for the experimental case of an array of annular holes drilled in a gold film, where the holes are filled with $\mathrm{SiO}_{2}$. Despite absorption losses in both $\mathrm{Au}$ and $\mathrm{SiO}_{2}$, the correct cutoff frequencies, $\omega_{+}$and $\omega_{-}$, are predicted by Eq. (S9). These cutoff frequencies are represented with vertical dashdotted lines in Fig. S4. Eq. (S9) works well in this case for the lossy response of the metal is already included in $\omega_{c_{\infty}}$, which is used as an input parameter. 


\section{S6 Phonon polariton branches in a coaxial waveguide, for a single vibrational mode}

The interaction of optical vibrational modes and the electromagnetic field is particularly important when frequencies and wavevectors of phonon and photon fields coincide near the crossover of the corresponding dispersion relations. The resulting phonon-polariton fields are the simultaneous solutions of Maxwell's equations (S4, S5) and lattice equations (S1, S2). These solutions are obtained from the Lagrangian density,

$$
\mathscr{L}=\frac{1}{8 \pi}\left(\varepsilon_{\infty} \mathbf{E}^{2}-\mathbf{H}^{2}\right)+\frac{1}{2}\left(\dot{\mathbf{x}}^{2}-\omega_{T O}^{2} \mathbf{x}^{2}\right)+\sqrt{\frac{\varepsilon_{\infty}}{4 \pi}} \omega_{p} \mathbf{x} \cdot \mathbf{E},
$$

using the Lagrangian equations. In this section, we compute the polaritonic branches of an infinite coaxial waveguide assuming that light interacts with a single vibrational mode with frequency $\omega_{T O}$ and coupling constant $\omega_{p}$. The electronic background of the ions is characterized by the high-frequency dielectric constant $\varepsilon_{\infty}$.

For the sake of convenience, the magnetic field is eliminated from Maxwell's equations (S4, S5) and the resulting wave equation (S6) is employed in the following. As dispersive terms of vibrational modes (proportional to the second derivative of the displacement field) are not included in Eq. (S10), the dynamical lattice equations derived with the Lagrangian equations coincide with those for the bulk (Eqs. S1, S2). The relative displacement field of the ions, $\mathbf{x}$, and the polarization field, $\mathbf{P}$, resulting from the lattice equations have the same symmetry as the electric field, $\mathbf{E}$, inside the waveguide and are parallel to it. This local relation between $\mathbf{x}, \mathbf{P}$, and $\mathbf{E}$ will be valid as long as the dispersion relation of the phonons is neglected, which is a good approximation because the $k$ dependence of the uncoupled optical phonons is negligible compared to that of photons.

Let us consider a given waveguide mode, $M$, characterized by a wavevector $k$ along the waveguide axis (for instance $M=T E_{11}$, the fundamental mode of the coaxial waveguide described in Sec. S2). When the aperture is filled with a material with a uniform dielectric constant $\varepsilon_{\infty}$, the electric field satisfies the wave equation (S6),

$$
\nabla \times \nabla \times E \mathbf{E}_{M}-\varepsilon_{\infty} \frac{\omega_{k}^{2}}{c^{2}} E \mathbf{E}_{M}=0,
$$

where $\mathbf{E}=E \mathbf{E}_{M}$ is the electric-field vector with amplitude $E$, and $\mathbf{E}_{M}$ is the normalized transverse solution of Maxwell's equations for mode $M$ at frequency $\omega_{k}$. The wave equation transforms to

$$
\nabla \times \nabla \times E \mathbf{E}_{M}-\frac{\omega^{2}}{c^{2}}(E+4 \pi P) \mathbf{E}_{M}=0
$$

in order to accommodate the polarization field, $\mathbf{P}=P \mathbf{E}_{M}$, of the oscillating ions when

the waveguide is filled with the phononic material. $\mathbf{E}_{M}$ satisfies both wave equations if 
the scaling relation (S8) is fulfilled; i.e., if

$$
\omega^{2}(E+4 \pi P)=\varepsilon_{\infty} \omega_{k}^{2} E .
$$

Sec. S5 shows that this is a good approximation for the coaxial hole array studied experimentally.

Both the relative displacement field and the polarization field for vibrational modes without spatial dispersion are determined by the mode profile of the electric field: $\mathbf{x}(\mathbf{r})=$ $x \mathbf{E}_{M}(\mathbf{r})$ and $\mathbf{P}(\mathbf{r})=P \mathbf{E}_{M}(\mathbf{r})$. Therefore, the lattice equations (S1, S2) may by written in terms of the field amplitudes $x, P$, and $E$ :

$$
\begin{aligned}
-\omega^{2} x & =-\omega_{T O}^{2} x+i \gamma \omega x+\sqrt{\frac{\varepsilon_{\infty}}{4 \pi}} \omega_{p} E, \\
P & =\sqrt{\frac{\varepsilon_{\infty}}{4 \pi}} \omega_{p} x+\frac{\varepsilon_{\infty}-1}{4 \pi} E,
\end{aligned}
$$

where a phenomenological damping force, $-\gamma \dot{x}$, has been added. Eliminating $P$ from equations (S11-S13), the system of equations can be expressed in a matrix form, stating that $\mathbf{E}_{M}(\mathbf{r}, \mathbf{k})$ is still a solution of Maxwell equations but at a frequency $\omega$ satisfying

$$
\left(\begin{array}{cc}
\omega^{2}-\omega_{T O}^{2}+i \gamma \omega & \omega \omega_{p} \\
\omega \omega_{p} & \omega^{2}-\omega_{k}^{2}+i \delta \omega_{k}
\end{array}\right) \cdot\left(\begin{array}{c}
\omega x \\
\sqrt{\varepsilon_{\infty} / 4 \pi} E
\end{array}\right)=0 .
$$

We have included a finite linewidth in the photon mode by making the substitution $\omega_{k} \rightarrow \omega_{k}-i \delta / 2$.

In the strong-coupling regime, one can assume that $\omega_{T O} \gg \omega_{p} \gg \gamma, \delta$ at the crossing point of the photon and TO phonon frequencies $\left(\omega_{k}=\omega_{T O}\right)$. We find from the secular equation (setting the determinant of the matrix equal to zero) that the degeneracy between the two modes is lifted:

$$
\omega_{ \pm}=\omega_{T O} \pm \frac{\omega_{p}}{2}-i \frac{\gamma_{F P}+\gamma}{4},
$$

and the frequency splitting, $\Delta \omega=\omega_{+}-\omega_{-}=\omega_{p}$, coincides with $\omega_{p}$ in this simple case of one phonon mode. Moreover, the width of each dressed state is the average of the photon $(\delta / 2)$ and phonon $(\gamma / 2)$ linewidths. The splitting can be resolved only if $\Delta \omega>(\gamma+\delta) / 2$.

In the USC regime, however, $\omega_{p}$ is no longer a small parameter, and we need to compute the exact values of the polaritonic frequencies at resonance,

$$
\omega_{ \pm}=\sqrt{\omega_{T O}^{2}+\frac{\omega_{p}^{2}}{4}} \pm \frac{\omega_{p}}{2}
$$



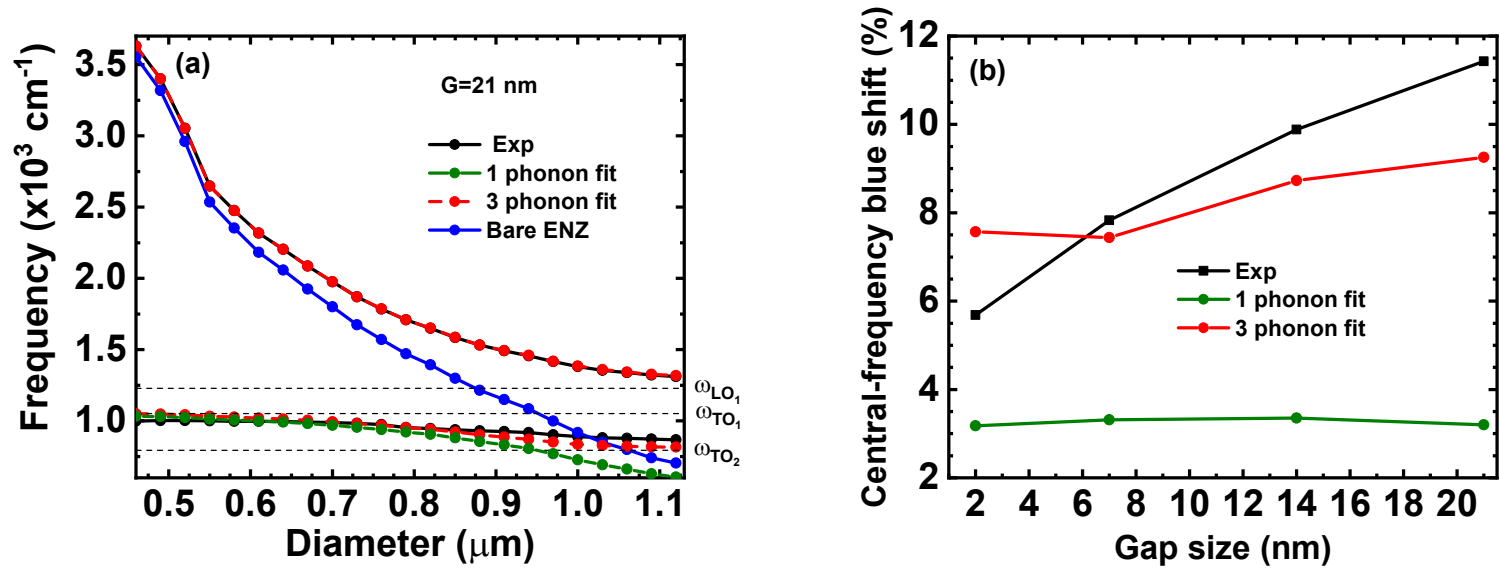

Figure S5. (a) Experimental transmission peak positions (black) for a coaxial nanocavity with a gap width of $21 \mathrm{~nm}$ filled with $\mathrm{SiO}_{2}$, and fitted values for 1 (green) and 3 (red) vibrational modes as well as bare ENZ modes (blue). (b) Blue shift, $\Delta \omega_{c e n}=\left(\omega_{c e n}-\omega_{T O_{1}}\right) / \omega_{T O_{1}}[\times 100 \%]$, of the central frequency, $\omega_{c e n}=\left(\omega_{+}+\omega_{-}\right) / 2$ at resonance $\left(\omega_{E N Z}=\omega_{T O_{1}}\right)$.

where absorption has been neglected for the sake of simplicity. We obtain the same splitting though the central frequency is blue-shifted from $\omega_{T O}$ to $\sqrt{\omega_{T O}^{2}+\omega_{p}^{2} / 4}$. An appreciable blue shifting of the central frequency is thus a signature of the USC regime. The experimental results reported in Fig. S5(b) show the expected blue shift of up to $11 \%$ of $\omega_{T O}$. This behaviour cannot be explained by the constant and smaller value of $3 \%$ predicted by the model that takes into account a single vibrational model of $\mathrm{SiO}_{2}$ (see Fig. S5(b)). Rather, experimental trends are reproduced only when several vibrational modes are taken into account (see Sec. S8).

Additional remarks:

- The frequency dependence of the off-diagonal coupling term is related to the longrange nature of the Coulomb forces (i.e. the dipole-dipole infraction induced via an electric field), and does not appear in the text-book example of two harmonic oscillators (Sec. S7), due to the short-range nature of the oscillator restoring forces.

- The Reststrahlen band is smaller than the splitting:

$$
\omega_{L 0}-\omega_{T O}=\left(\omega_{p} / 2 \omega_{T O}\right) \omega_{p}<\Delta \omega
$$

and thus, the splitting is not simply the manifestation of absorption induced within the Reststrahlen band. 
- The asymptotic limits of the general solution are

$$
\begin{aligned}
\lim _{\omega_{k}^{\prime} \rightarrow 0} \omega_{-} & =\omega_{k} \sqrt{\varepsilon_{\infty} / \varepsilon_{0}}, & \text { photon-like } \\
\lim _{\omega_{k}^{\prime} \rightarrow 0} \omega_{+}=\omega_{L O}, & & \text { phonon-like } \\
\lim _{\omega_{k}^{\prime} \rightarrow \infty} \omega_{-}=\omega_{T O}, & & \text { phonon-like } \\
\lim _{\omega_{k}^{\prime} \rightarrow \infty} \omega_{+}=\omega_{k} . & & \text { photon-like }
\end{aligned}
$$

The existence of 4 different asymptotes is a clear difference with respect to the case of two harmonic oscillators coupled with short-range interactions, which present only three asymptotic values (as the asymptotes represented by $\omega_{T O}$ and $\omega_{L O}$ would coincide in this case).

- The vibrational coupling constant is a function of the molecular concentration $n$, according to $\omega_{p}=\sqrt{4 \pi n e^{2} / M}$ (Eq. S3). The dependence $\omega_{p} \sim \sqrt{n}$ is shared by classical, semi-classical and quantum descriptions ${ }^{4}$. This behaviour has been confirmed experimentally for a classical Fabry-Perot resonator ${ }^{5}$.

\section{S7 Coupled harmonic oscillators}

We consider two mechanical oscillators with eigenfrequencies $\sqrt{k_{A} / m_{A}}$ and $\sqrt{k_{B} / m_{B}}$ coupled by a spring with constant $\kappa$ as a canonical model for strong coupling ${ }^{6}$. The differential equations

$$
\begin{aligned}
& m_{A} \ddot{x}_{A}+k_{A} x_{A}+\kappa\left(x_{A}-x_{B}\right)=0, \\
& m_{B} \ddot{x}_{B}+k_{B} x_{B}-\kappa\left(x_{A}-x_{B}\right)=0,
\end{aligned}
$$

describe the motion of the system. The harmonic solutions of these equations can be written in the matrix form

$$
\left(\begin{array}{cc}
\omega^{2}-\omega_{A}^{2} & \Gamma \\
\Gamma & \omega^{2}-\omega_{B}^{2}
\end{array}\right) \cdot\left(\begin{array}{c}
\sqrt{m_{A}} x_{A} \\
\sqrt{m_{B}} x_{B}
\end{array}\right)=0,
$$

where $\omega_{A}^{2}=\left(k_{A}+\kappa\right) / m_{A}, \omega_{B}^{2}=\left(k_{B}+\kappa\right) / m_{B}$, and $\Gamma=\kappa / \sqrt{m_{A} m_{B}}$. The diagonal terms contain the eigenfrequencies of the uncoupled oscillators, modified by their interaction, while the off-diagonal terms are proportional to the coupling strength. The secular equation yields the dressed frequencies of the system.

$$
\omega_{ \pm}^{2}=\frac{\omega_{A}^{2}+\omega_{B}^{2} \pm \sqrt{\left(\omega_{A}^{2}-\omega_{B}^{2}\right)^{2}+4 \Gamma^{2}}}{2} .
$$

The frequency splitting, $\omega_{+}-\omega_{-} \approx \Gamma$, increases with the coupling constant. A numerical example is presented in Fig. S6. 


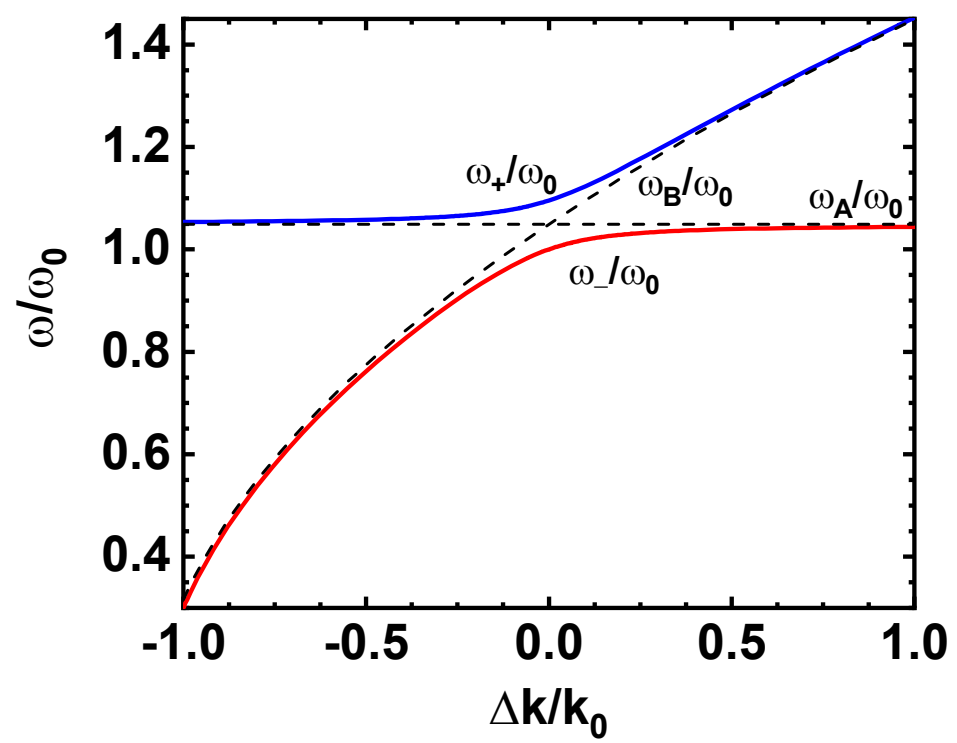

Figure S6. Bare (dashed lines) and dressed (solid lines) frequencies for a pair of coupled harmonic oscillators with $\omega_{A}=\omega_{0} \sqrt{1+\kappa / k_{0}}, \omega_{B}=\omega_{0} \sqrt{1+\Delta k / k_{0}+\kappa / k_{0}}$, and $\kappa=0.1 k_{0}$.

\section{S8 Multiple vibrational degrees of freedom}

Sec. S6 discusses polaritonic branches in a coaxial waveguide filled with a material with a single vibrational optical mode. We consider now the influence of additional vibrational modes, with damping, in the optical response of the filled coaxial aperture. The lattice equations of motion for a unit cell with $N$ oscillator normal modes are

$$
\begin{aligned}
\ddot{x}_{1} & =-\omega_{T O_{1}}^{2} x_{1}-\gamma_{1} \dot{x}_{1}+\sqrt{\frac{\varepsilon_{\infty}}{4 \pi}} \omega_{p_{1}} E, \\
\ddot{x}_{2} & =-\omega_{T O_{2}}^{2} x_{2}-\gamma_{2} \dot{x}_{2}+\sqrt{\frac{\varepsilon_{\infty}}{4 \pi}} \omega_{p_{2}} E, \\
& \vdots \\
\ddot{x}_{N} & =-\omega_{T O_{N}}^{2} x_{N}-\gamma_{N} \dot{x}_{N}+\sqrt{\frac{\varepsilon_{\infty}}{4 \pi}} \omega_{p_{N}} E, \\
P & =\sqrt{\frac{\varepsilon_{\infty}}{4 \pi}} \sum_{i=1}^{N} \omega_{p_{i}} x_{i}+\frac{\varepsilon_{\infty}-1}{4 \pi} E .
\end{aligned}
$$


Simultaneous solution of the lattice and Maxwell's equations for transverse modes can be expressed as a matrix equation of order $N+1$ :

$$
\left(\begin{array}{ccccc}
\omega^{2}-\omega_{T O_{1}}^{2}+i \gamma_{1} \omega & 0 & \ldots & 0 & \omega \omega_{p_{1}} \\
0 & \omega^{2}-\omega_{T O_{2}}^{2}+i \gamma_{2} \omega & \ldots & 0 & \omega \omega_{p_{2}} \\
\vdots & \vdots & \vdots & \vdots & \vdots \\
0 & 0 & \ldots & \omega^{2}-\omega_{T O_{N}}^{2}+i \gamma_{N} \omega & \omega \omega_{p_{N}} \\
\omega \omega_{p_{1}} & \omega \omega_{p_{2}} & \ldots & \omega \omega_{p_{N}} & \omega^{2}-\omega_{k}^{\prime 2}
\end{array}\right) \cdot \mathbf{F}=0
$$

where $\mathbf{F}=\left(\omega x_{1}, \omega x_{2}, \ldots, \omega x_{N}, \sqrt{\varepsilon_{\infty} / 4 \pi} E\right)^{t}$. The secular equation for this matrix can be written as

$$
\varepsilon(\omega) \omega^{2}=\varepsilon_{\infty} \omega_{k}^{2},
$$

provided that the effective dielectric function is generalized to include all vibrational modes:

$$
\varepsilon(\omega)=\varepsilon_{\infty}\left(1+\sum_{j=1}^{N} \frac{\omega_{p_{j}}^{2}}{\omega_{T O_{j}}^{2}-\omega^{2}-i \gamma_{j} \omega}\right) .
$$

In Fig. S5(a), the experimental spectral position of transmission resonances for an array of coaxial holes filled with $\mathrm{SiO}_{2}$ are compared with those calculated with the formalism described above. Calculations were done for two models for the dielectric constant of $\mathrm{SiO}_{2}$ : in one of them we assumed that only one vibrational mode contributed to $\varepsilon_{\mathrm{SiO}_{2}}$, while in the other we considered 3 vibrational modes (the contribution from each vibrational mode to $\varepsilon_{\mathrm{SiO}_{2}}$ is described by a Lorentzian function using Palik's data ${ }^{7}$ ). For a single vibrational mode, we find a good agreement with the upper band, but the lower band is poorly described. The agreement with both bands is improved when two lowerfrequency vibrational modes are added. Note that the size of the main gap is reduced in this case.

Fig. S5(b) shows the blue shifting, $\Delta \omega_{c e n}=\left(\omega_{c e n}-\omega_{T O_{1}}\right) / \omega_{T O_{1}}[\times 100 \%]$, of the central frequency, $\omega_{\text {cen }}=\left(\omega_{+}+\omega_{-}\right) / 2$, computed from the experimental values of the upper $\left(\omega_{+}\right)$and lower $\left(\omega_{-}\right)$polaritonic frequencies at resonance as a function of the experimental gap sizes, $G=2,7,14$, and $21 \mathrm{~nm}$. $\Delta \omega_{\text {cen }}$ increases from $6 \%$ at $G=2 \mathrm{~nm}$ to $11 \%$ at $G=21 \mathrm{~nm}$. This behaviour cannot be explained by the constant value of $3 \%$ predicted by our model if we assume that a single vibrational model of $\mathrm{SiO}_{2}$ is interacting with the cavity photons. A better agreement is obtained when 3 vibrational modes are taken into account, see Fig. S5(b). Therefore, we conclude that the interaction with several vibrational modes reduces the gap size of the main gap but increases the blue shifting of its central frequency. 


\section{S9 Quantum theory of phonon polaritons}

In this section, we present the quantum-mechanical treatment of the coupled photon and phonon modes in a coaxial cavity of length $L$ with ideal PEC walls. Such simplified boundary conditions cannot provide a good quantitative agreement with the polaritonic frequencies experimentally measured for gap sizes smaller than the skin depth. However, the qualitative results derived here validate the classical approach of Sec. S6.

We follow the canonical procedure for second quantization and write a Hopfield-like Hamiltonian in the representation of the number operators for photons and phonons. We start from the classical Lagrangian of the system under study, which is the integral of the Lagrangian density (Eq. S10) previously used for computing the classical equations of motion:

$$
L=\int\left[\frac{1}{8 \pi}\left(\frac{\varepsilon_{\infty}}{c^{2}} \dot{\mathbf{A}}^{2}-(\nabla \times \mathbf{A})^{2}\right)+\frac{1}{2}\left(\dot{\mathbf{x}}^{2}-\omega_{T O}^{2} \mathbf{x}^{2}\right)-\frac{\gamma_{12}}{c} \mathbf{x} \cdot \dot{\mathbf{A}}\right] d^{3} r
$$

where, for the sake of convenience, electric and magnetic fields have been expressed as a function of the vector potential $\mathbf{A}$ (in the Coulomb gauge, $\nabla \cdot \mathbf{A}=0$ ); i.e., $\mathbf{E}=$ $\dot{\mathbf{A}} / c, \mathbf{H}=\nabla \times \mathbf{A} . \quad \mathbf{A}=A \mathbf{A}_{m}(\mathbf{r})$ is a solution of Maxwell's wave equations with the appropriate boundary conditions for a given mode $m, A$ is the field amplitude, and $\mathbf{A}_{m}(\mathbf{r})$ the mode profile. The mode is defined as $m=\left\{M, k_{z}\right\}$, where $M$ takes into account both polarization and field profile in the $X Y$ plane and, along the direction perpendicular to the plane, $k_{z}=\pi \ell / L$, with $\ell=1,2,3, \ldots$. In the numerical calculations below, we will consider only the fundamental $M=\mathrm{TE}_{11}$ mode for a given $k_{z}$. As we assume a non-dispersive vibrational mode with frequency $\omega_{T O}$, the relative displacement field is proportional to the vector potential inside the cavity, $\mathbf{x}=x \mathbf{A}_{m}(\mathbf{r}) \cdot \gamma_{12}^{2}=\varepsilon_{\infty} \omega_{p}^{2} / 4 \pi$ is the classical coupling constant.

Following Hopfield ${ }^{8}$, the Lagrangian $L$ can be transformed to

$$
L^{\prime}=L+\int \frac{\gamma_{12}}{c} \frac{\partial(\mathbf{x} \cdot \mathbf{A})}{\partial t} d^{3} r
$$

The Hamiltonian of the system is derived from the Lagrangian function with help of the canonical momentum fields, $\mathbf{B}=\partial \mathscr{L} / \partial \dot{\mathbf{A}}=\varepsilon_{\infty} \dot{\mathbf{A}} / 4 \pi c^{2}$ and $\mathbf{Y}=\partial \mathscr{L} / \partial \dot{\mathbf{x}}=\dot{\mathbf{x}}+\gamma_{12} \mathbf{A} / c$, 
and the canonically conjugate operators,

$$
\begin{aligned}
\hat{\mathbf{A}} & =\sum_{m} \sqrt{\frac{2 \pi \hbar c^{2}}{V_{m} \omega_{m} \varepsilon_{\infty}}} \mathbf{A}_{m}(\mathbf{r})\left(a_{m}+a_{m}^{+}\right), \\
\hat{\mathbf{B}} & =i \sum_{m} \sqrt{\frac{\hbar \omega_{m} \varepsilon_{\infty}}{8 \pi c^{2} V_{m}}} \mathbf{A}_{m}(\mathbf{r})\left(a_{m}^{+}-a_{m}\right), \\
\hat{\mathbf{x}} & =\sum_{m} \sqrt{\frac{\hbar}{2 V_{m} \omega_{T O}}} \mathbf{A}_{m}(\mathbf{r})\left(b_{m}+b_{m}^{+}\right), \\
\hat{\mathbf{Y}} & =i \sum_{m} \sqrt{\frac{\hbar \omega_{T O}}{2 V_{m}}} \mathbf{A}_{m}(\mathbf{r})\left(b_{m}^{+}-b_{m}\right),
\end{aligned}
$$

where $\omega_{m}$ is the photon frequency, $a^{+}(a)$ and $b^{+}(b)$ are the creation (annihilation) operators for phonons and photons, respectively, and $V_{m}$ is the mode volume for both fields. These operators produce the Hamiltonian in the new representation

$$
H=H_{\text {photon }}+H_{\text {phonon }}+H_{\text {int }},
$$

with

$$
\begin{aligned}
H_{\text {photon }}= & \sum_{m} \hbar \omega_{m}\left(a_{m}^{+} a_{m}+\frac{1}{2}\right), \\
H_{\text {phonon }}= & \sum_{m} \hbar \omega_{T O}\left(b_{m}^{+} b_{m}+\frac{1}{2}\right), \\
H_{\text {int }}= & \sum_{m} \hbar\left[i C_{m}\left(a_{m}^{+} b_{m}-a_{m} b_{m}^{+}\right)+D_{m}\left(2 a_{k}^{+} a_{m}+1\right)\right. \\
& \left.+i C_{m}\left(a_{m} b_{m}-a_{m}^{+} b_{m}^{+}\right)+D_{m}\left(a_{m} a_{m}+a_{m}^{+} a_{m}^{+}\right)\right] .
\end{aligned}
$$

$H_{\text {photon }}$ and $H_{\text {phonon }}$ describe the energy of bare cavity photons and phonons in terms of their respective number operators $a_{m}^{+} a_{m}$ and $b_{m}^{+} b_{m}$. Several terms contribute to $H_{\text {int }}$ that depend on the coupling constants

$$
C_{m}=\frac{\omega_{p}}{2} \sqrt{\frac{\omega_{T O}}{\omega_{m}}}, D_{m}=\frac{\omega_{p}^{2}}{4 \omega_{m}} .
$$

Terms in the first line constitute the resonant part of the light-matter interaction ${ }^{9}$. The term proportional to $C_{m}$ describes the creation (annihilation) of one photon and the annihilation (creation) of a phonon with the same wave number $m$. The term proportional to $D_{m}$ comes from the $\mathbf{A}^{2}$ term in the original Hamiltonian. It contains the photon number operator that produces a blue shifting (as $D_{m}>0$ ) of the bare cavity phonon frequency. In the second line of $H_{i n t}$, we find the antiresonant terms that are frequently 
neglected when the Hamiltonian is diagonalized. These terms produce the simultaneous destruction or creation of two excitations.

We will diagonalize the full Hamiltonian (including the antiresonant terms) using the Bogoliubov transformation,

$$
p_{m i}=w_{m i} a_{m}+x_{m i} b_{m}+y_{m i} a_{m}^{+}+z_{m i} b_{m}^{+} .
$$

The Bose commutation rule $\left[p_{m i}, p_{m^{\prime} i^{\prime}}^{+}\right]=\delta_{i, i^{\prime}} \delta_{m, m^{\prime}}$, where $i, i^{\prime}$ are two solutions of the secular equation, imposes the normalization condition,

$$
w_{m i}^{*} w_{m i^{\prime}}+x_{m i}^{*} x_{m i^{\prime}}-y_{m i}^{*} y_{m i^{\prime}}-z_{m i}^{*} z_{m i^{\prime}}=\delta_{i, i^{\prime}},
$$

to the Hopfield coefficients. From the equation of motion for operators in the Heisenberg picture,

$$
\left[p_{m i}, \hat{H}\right]=\hbar \omega p_{m i}
$$

the eigenvalue problem may be written in a matrix form:

$$
\left(\begin{array}{cccc}
\omega_{m}+2 D_{m}-\omega & -i C_{m} & -2 D_{m} & -i C_{m} \\
i C_{m} & \omega_{T O}-\omega & -i C_{m} & 0 \\
2 D_{m} & -i C_{m} & -\omega_{m}-2 D_{m}-\omega & -i C_{m} \\
-i C_{m} & 0 & i C_{m} & -\omega_{T O}-\omega
\end{array}\right)\left(\begin{array}{c}
w_{m i} \\
x_{m i} \\
y_{m i} \\
z_{m i}
\end{array}\right)=0 .
$$

The determinant of the matrix, $\omega^{4}-\left(\omega_{m}^{2}+\omega_{T O}^{2}+\omega_{p}^{2}\right) \omega^{2}+\omega_{m}^{2} \omega_{T O}^{2}=0$, is identical to the one obtained with the classical approach above (if absorption is neglected). In other words, quantum and classical descriptions provide the same polaritonic branches. This equation may therefore be rewritten in the form $\varepsilon(\omega) \omega^{2}=\varepsilon_{\infty} \omega_{m}^{2}$, where $\varepsilon(\omega)=$ $\varepsilon_{\infty}\left[1+\omega_{p}^{2} /\left(\omega_{T O}^{2}-\omega^{2}\right)\right]$.

In Fig. S7(a) the measured polaritonic frequencies are fitted with our model assuming that the fundamental cavity photon is interacting with the TO phonon. The corresponding Hopfield coefficients $|w-y|^{2}$ and $|x-z|^{2}$, which are solutions of the matrix equation for a given eigenvalue, are depicted in Fig. S7(b). The Hopfield coefficients evaluate the relative contribution of photon and phonon modes to the polaritonic states.

The quantum approach accounts for the content of bare modes in the ground state energy. The ground state of polariton excitations, $|G\rangle$, contains a finite number of virtual cavity photons and phonons per mode ${ }^{9}$,

$$
\begin{aligned}
\left\langle G\left|a_{m}^{+} a_{m}\right| G\right\rangle & =\sum_{i= \pm}\left|y_{i m}\right|^{2}, \\
\left\langle G\left|b_{m}^{+} b_{m}\right| G\right\rangle & =\sum_{i= \pm}\left|z_{i m}\right|^{2},
\end{aligned}
$$



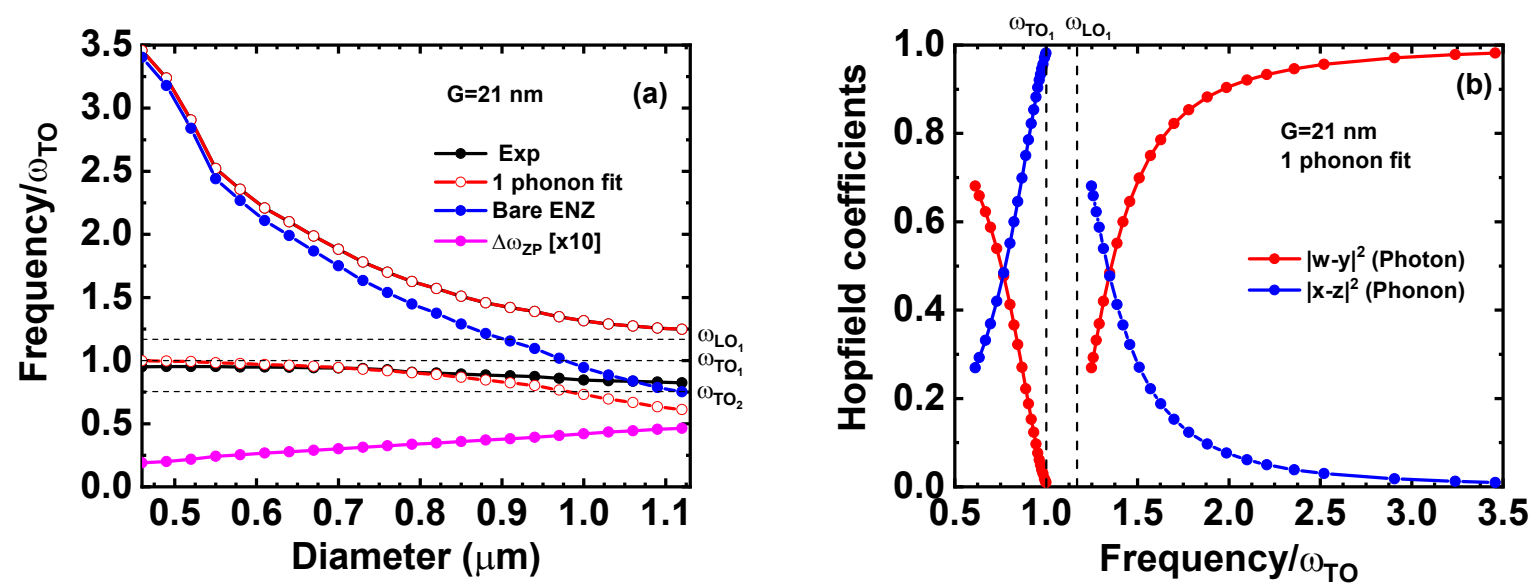

Figure S7. (Colour online). (a) Measured phonon polariton branches and the calculated zero-point differential frequency, $\Delta \omega_{Z P}[x 10]$. Experimental points are fitted with the eigenfrequencies calculated assuming that cavity photons interact with a single vibrational mode of $\mathrm{SiO}_{2}$. The bare ENZ frequency is the single fitting parameter. (b) Hopfield coefficients corresponding to the measured frequencies.

where $i= \pm$ for the two polaritonic branches. The difference between the ground state energy of the polaritons, $E_{G}$, and ground state energy of the uncoupled system, $E_{0}$, per mode

$$
E_{G}-E_{0}=\hbar \Delta \omega_{Z P}=\hbar D_{m}-\sum_{i= \pm} \hbar \omega_{i}\left(\left|y_{i m}\right|^{2},+\left|z_{i m}\right|^{2}\right),
$$

is a function of the number of virtual modes ${ }^{10}$. We find in the first term of this expression the positive coupling constant, $D_{m}$, and in the second term the negative correlation contribution for a given mode, $\hbar \Delta \omega_{Z P}^{c o r}$, for the two polaritonic frequencies $\omega_{ \pm}{ }^{9}$. These quantities can be written as a function of the normalized coupling strength $\eta=\omega_{p} / 2 \omega_{T O}$ at resonance $\left(\omega_{m}=\omega_{T O}\right)$ if we use the exact value of the polaritonic frequencies, $\omega_{ \pm} / \omega_{T O}=\sqrt{1+\eta^{2}} \pm \eta$, and retain the leading terms in the series expansion for both the positive coupling constant $D_{m}$ and the Hopfield coefficients:

$$
\begin{aligned}
\frac{D_{m}}{\omega_{T O}} & =\eta^{2}, \\
\frac{\Delta \omega_{Z P}^{c o r}}{\omega_{T O}} & =-\frac{\eta^{2}}{2} \sqrt{1+\eta^{2}} .
\end{aligned}
$$

Calculated values of these quantities and the resulting zero-point differential frequency, $\Delta \omega_{Z P}=D_{m}-\Delta \omega_{Z P}^{c o r}$, are reported in Table S2 for measured values of $\eta$. We find that $\Delta \omega_{Z P}$ is about $3 \%$ of the resonance frequency, $\omega_{T O}$. Moreover, Fig. S7 (a) compares the polaritonic branches with the exact differential zero point frequency $\Delta \omega_{Z P}$, which increases from $2 \%$ of $\omega_{T O}$ at diameter $D=460 \mathrm{~nm}$ to $5 \%$ of $\omega_{T O}$ at $D=1120 \mathrm{~nm}$. 


\begin{tabular}{c|c|c|c|c}
\hline \hline $\mathrm{SiO}_{2}$ gap $(\mathrm{nm})$ & 2 & 7 & 14 & 21 \\
\hline$\eta$ & 0.254 & 0.26 & 0.261 & 0.255 \\
$D_{m} / \omega_{T O}(\%)$ & 6.45 & 6.73 & 6.81 & 6.50 \\
$\Delta \omega_{Z P}^{\text {cor }} / \omega_{T O}(\%)$ & -3.33 & -3.48 & -3.52 & -3.35 \\
\hline$\Delta \omega_{Z P} / \omega_{T O}(\%)$ & 3.12 & 3.25 & 3.29 & 3.15 \\
\hline \hline
\end{tabular}

Table S2. Differential zero-point frequency, $\Delta \omega_{Z P}=D_{m}+\Delta \omega_{Z P}^{c o r}$, at resonance, $\omega_{k}=\omega_{T O}$, for the measured normalized coupling strength, $\eta$. The negative correlation contribution, $\Delta \omega_{Z P}^{c o r}$, and the positive coupling coefficient, $D_{m}$, are also reported. These quantities are normalized by the resonance frequency $\omega_{T O}$.

\section{S10 Hopfield-like Hamiltonian for multiple vibrational degrees of freedom}

The Hopfield Hamiltonian can be easily generalized to include $N$ vibrational modes:

$$
\begin{aligned}
H_{\text {photon }}= & \sum_{m} \hbar \omega_{m}\left(a_{m}^{+} a_{m}+\frac{1}{2}\right), \\
H_{\text {phonon }}= & \sum_{j m} \hbar \omega_{T O_{j}}\left(b_{j m}^{+} b_{j m}+\frac{1}{2}\right), \\
H_{\text {int }}= & \sum_{j m} \hbar\left[i C_{j m}\left(a_{m}^{+} b_{j m}-a_{m} b_{j m}^{+}\right)+D_{j m}\left(2 a_{k}^{+} a_{m}+1\right)\right. \\
& \left.+i C_{j m}\left(a_{m} b_{j m}-a_{m}^{+} b_{j m}^{+}\right)+D_{j m}\left(a_{m} a_{m}+a_{m}^{+} a_{m}^{+}\right)\right],
\end{aligned}
$$

with $j=1, \ldots, N$. A new Bogoliubov transformation,

$$
p_{m i}=w_{m i} a_{m}+\sum_{j} x_{j m i} b_{j m}+y_{m i} a_{m}^{+}+\sum_{j} z_{j m i} b_{j m}^{+},
$$

is used to diagonalize the Hamiltonian. The eigenvalue problem may be expressed as a $2(N+1) \times 2(N+1)$ matrix,

$$
\left(\begin{array}{ccccccc}
\omega_{m}+2 D_{m}-\omega & -2 D_{m} & -i C_{1 m} & -i C_{1 m} & \cdots & -i C_{N m} & -i C_{N m} \\
2 D_{m} & -\omega_{m}-2 D_{m}-\omega & -i C_{1 m} & -i C_{1 m} & \cdots & -i C_{N m} & -i C_{N m} \\
i C_{1 m} & -i C_{1 m} & \omega_{T O_{1}}-\omega & 0 & \cdots & 0 & 0 \\
-i C_{1 m} & i C_{1 m} & 0 & -\omega_{T O_{1}}-\omega & \cdots & 0 & 0 \\
\vdots & \vdots & \vdots & \vdots & \vdots & \vdots & \vdots \\
i C_{N m} & -i C_{N m} & 0 & 0 & \cdots & \omega_{T O_{N}}-\omega & 0 \\
-i C_{N m} & i C_{N m} & 0 & 0 & \cdots & 0 & -\omega_{T O_{N}}-\omega
\end{array}\right) \cdot \mathbf{F}=0 .
$$

where $\mathbf{F}=\left(w_{m i}, y_{m i}, x_{1 m i}, z_{1 m i}, \ldots, x_{N m i}, z_{N m i}\right)^{t}$ is a column vector, and

$$
\begin{aligned}
D_{m} & =\sum_{i=1}^{N} D_{i m}=\frac{1}{4 \omega_{m}} \sum_{i=1}^{N} \omega_{p_{i}}^{2}, \\
C_{i m} & =\frac{\omega_{p_{i}}}{2} \sqrt{\frac{\omega_{T O_{i}}}{\omega_{m}}},
\end{aligned}
$$

are the coupling constants. The secular equation may be also written as in the classical approach, $\omega^{2} \varepsilon(\omega)=\varepsilon_{\infty} \omega_{k}^{2}$, where the dielectric constant $\varepsilon(\omega)$ is a sum of several Lorentzian functions (Eq. S15). 

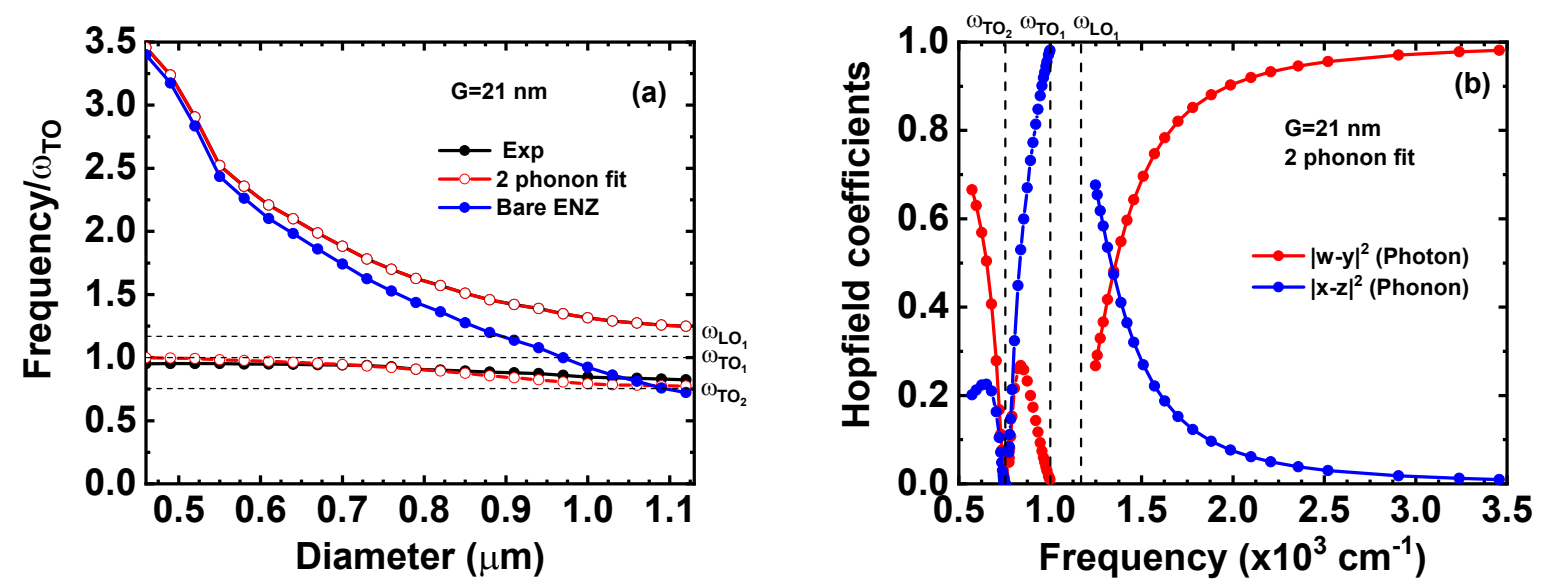

Figure S8. (Colour online). (a) Measured phonon polariton branches fitted with the eigenfrequencies calculated assuming that cavity photons interact with two vibrational modes of $\mathrm{SiO}_{2}$. The bare ENZ frequency is the single fitting parameter. (b) Hopfield coefficients corresponding to the measured frequencies.

Employing the normalization condition,

$$
w_{m i}^{*} w_{m i^{\prime}}+\sum_{j} x_{j m i}^{*} x_{j m i^{\prime}}-y_{m i}^{*} y_{m i^{\prime}}-\sum_{j} z_{j m i}^{*} z_{j m i i^{\prime}}=\delta_{i, i^{\prime}}
$$

we find the final expression for the normalized Hopfield coefficients for the eigenvalue $\omega_{i}$ of the $i^{t h}$ polaritonic branch,

$$
\begin{aligned}
& w_{m i}=\frac{1}{D}\left(1-\frac{\omega_{i}^{2}}{\omega_{T O_{1}}^{2}}\right)\left(\frac{\omega_{m}}{\omega_{T O_{1}}}+\frac{\omega_{i}}{\omega_{T O_{1}}}\right), \\
& x_{j m i}=-\frac{i}{D} \frac{1-\frac{\omega_{i}}{\omega_{T O_{1}}}}{1-\frac{\omega_{i}}{\omega_{T O_{j}}}} \frac{\omega_{p_{i}}}{\omega_{T O_{j}}} \sqrt{\frac{\omega_{T O_{i}} \omega_{m}}{\omega_{T O_{1}}^{2}}}\left(1+\frac{\omega_{i}}{\omega_{T O_{1}}}\right), \\
& y_{m i}=-\frac{1}{D}\left(1-\frac{\omega_{i}^{2}}{\omega_{T O_{1}}^{2}}\right)\left(\frac{\omega_{m}}{\omega_{T O_{1}}}-\frac{\omega_{i}}{\omega_{T O_{1}}}\right), \\
& z_{j m i}=-\frac{i}{D} \frac{1+\frac{\omega_{i}}{\omega_{T O_{1}}}}{1+\frac{\omega_{i}}{\omega_{T O_{j}}}} \frac{\omega_{p_{i}}}{\omega_{T O_{j}}} \sqrt{\frac{\omega_{T O_{i}} \omega_{m}}{\omega_{T O_{1}}^{2}}}\left(1-\frac{\omega_{i}}{\omega_{T O_{1}}}\right),
\end{aligned}
$$

which share the denominator

$$
D=2\left[\frac{\omega_{m} \omega_{i}}{\omega_{T O_{1}}^{2}}\left(\frac{\omega_{p_{1}}^{2}}{\omega_{T O_{1}}^{2}}+\left(1-\frac{\omega_{i}^{2}}{\omega_{T O_{1}}^{2}}\right)^{2}+\sum_{j>1} \frac{\omega_{p_{j}}^{2}}{\omega_{T O_{j}}^{2}} \frac{\left(1-\frac{\omega_{i}^{2}}{\omega_{T O_{1}}^{2}}\right)^{2}}{\left(1-\frac{\omega_{i}^{2}}{\omega_{T O_{j}}^{2}}\right)^{2}}\right)\right]^{1 / 2} .
$$


We have highlighted the relevance of the first vibrational mode for the experiments. All frequencies are normalized by $\omega_{T O_{1}}$. In Fig. S8(a) the measured polaritonic frequencies are fitted with our model assuming that two vibrational modes interact with the cavity photon. The corresponding Hopfield coefficients $|w-y|^{2}$ and $|x-z|^{2}$, depicted in Fig. S8(b), evaluate the relative contribution of photon and phonon modes to the polaritonic states.

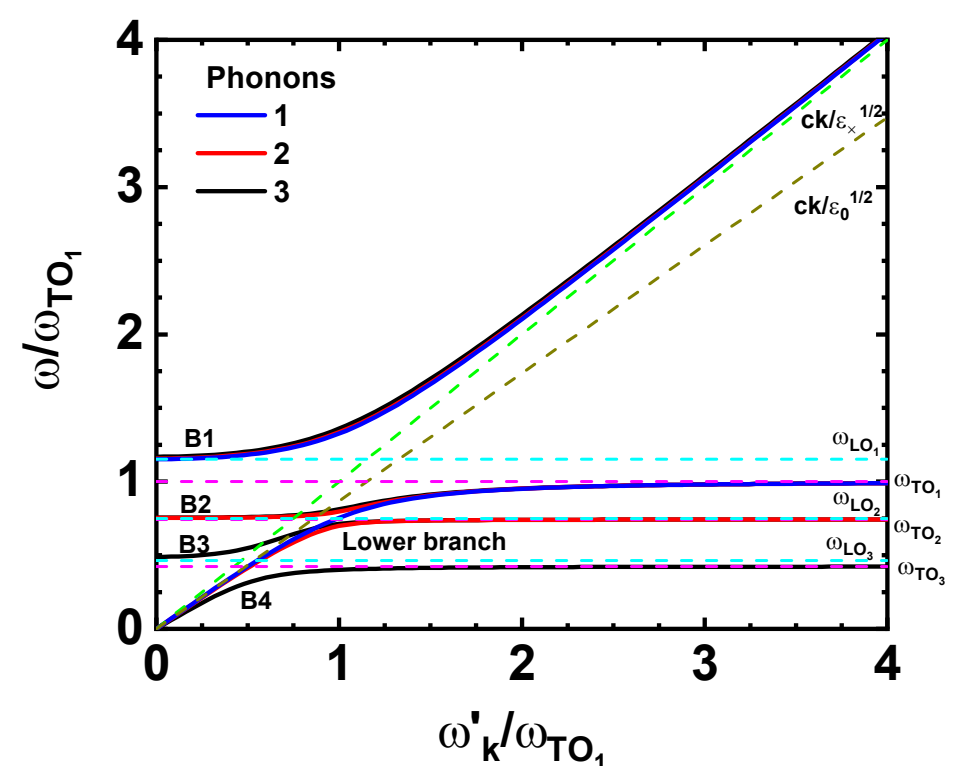

Figure S9. Dispersion relation of phonon polaritons for the vibrational modes of bulk $\mathrm{SiO}_{2}$. 


\section{S11 Bulk phonon polaritons}

Transverse waves in a homogeneous material also satisfy the propagation condition $\varepsilon(\omega) \omega^{2}=\omega_{k}^{2}$, where $\omega_{k}=c k, c$ is the speed of light and $k$ the wavenumber of the plain wave. Following the approach outlined in Sec. S6, it is straightforward to show that bulk polaritonic modes are solution of the matrix equation (S14). Fig. S9 shows the polaritonics bands in bulk $\mathrm{SiO}_{2}$ for three vibrational modes.

\section{S12 Fitting experimental data to the cavity model}

For a given coaxial array filled with a phononic material, let us consider we have the spectral positions of the transmission resonances, $\omega_{i}$, for the $N+1$ polaritonic branches corresponding to the $N$ vibrational modes and the fundamental waveguide mode. The fitting to the polaritonic dispersion relation, $\omega^{2} \varepsilon(\omega)=\varepsilon_{\infty} \omega_{k}^{2}$, consists in the following two steps:

1. Extract the dispersion relation of the unfilled hole from the spectral positions, $\omega_{k}=$ $\omega_{i_{0}} \sqrt{\varepsilon\left(\omega_{i_{0}}\right) / \varepsilon_{\infty}}$. Notice that a single branch $i_{0}$ is needed.

2. Solve the polynomial equation $\omega^{2} \varepsilon(\omega)=\varepsilon_{\infty} \omega_{k}$ for the remaining values of $i$ in order to obtain the fitting frequencies.

Fig. S5(a) shows the results of the fitting for a coaxial gap of $21 \mathrm{~nm}$ filled with $\mathrm{SiO}_{2}$. Fitting with one and three vibrational modes are compared with experimental results. 


\section{References}

1. García-Vidal, F. J., Martín-Moreno, L., Ebbesen, T. W. \& Kuipers, L. Light passing through subwavelength apertures. Rev. Mod. Phys. 82, 729-787, DOI: 10.1103/ RevModPhys.82.729 (2010).

2. Alù, A. \& Engheta, N. Light squeezing through arbitrarily shaped plasmonic channels and sharp bends. Phys. Rev. B 78, 035440, DOI: 10.1103/PhysRevB.78.035440 (2008).

3. Born, M. \& Huang, K. Dynamical theory of crystal lattices (Oxford University Press, 1988).

4. Törmä, P. \& Barnes, W. L. Strong coupling between surface plasmon polaritons and emitters: a review. Rep. Prog. Phys. 78, 013901, DOI: 10.1088/0034-4885/78/ 1/013901 (2015).

5. Zhu, Y. et al. Vacuum Rabi splitting as a feature of linear-dispersion theory: Analysis and experimental observations. Phys. Rev. Lett. 64, 2499-2502, DOI: 10.1103/PhysRevLett.64.2499 (1990).

6. Novotny, L. \& Hecht, B. Principles of Nano-optics (Cambridge University Press, New York, 2006).

7. Palik, E. D. Handbook of Optical Constants of Solids (Academic, London, 1985).

8. Hopfield, J. J. Theory of the contribution of excitons to the complex dielectric constant of crystals. Phys. Rev. 112, 1555-1567, DOI: 10.1103/PhysRev.112.1555 (1958).

9. Ciuti, C., Bastard, G. \& Carusotto, I. Quantum vacuum properties of the intersubband cavity polariton field. Phys. Rev. B 72, 115303, DOI: 10.1103/PhysRevB.72. 115303 (2005).

10. Quattropani, A., Andreani, L. C. \& Bassani, F. Quantum theory of polaritons with spatial dispersion: Exact solutions. Il Nuovo Cimento D 7, 55-69, DOI: 10.1007/ BF02452395 (1986). 